\title{
The Kondo screening cloud: what it is and how to observe it
}

\author{
Ian Affleck \\ Department of Physics and Astronomy, University of British Columbia, Vancouver, BC, Canada V6T $1 Z 1$
}

\begin{abstract}
The Kondo effect involves the formation of a spin singlet by a magnetic impurity and conduction electrons. It is characterized by a low temperature scale, the Kondo temperature, $T_{K}$, and an associated long length scale, $\xi_{K} \equiv \hbar v_{F} /\left(k_{B} T_{K}\right)$ where $v_{F}$ is the Fermi velocity. This Kondo length is often estimated theoretically to be in the range of .1 to 1 microns but such a long characteristic length scale has never been observed experimentally. In this review, I will examine how $\xi_{K}$ appears as a crossover scale when one probes either the dependence of physical quantities an distance from the impurity or when the impurity is embedded in a finite size structure and discuss possible experiments that might finally observe this elusive length scale.
\end{abstract}

\section{INTRODUCTION}

The Kondo model gives a simplified description of the interaction of a single magnetic impurity with the conduction electrons in a host, with Hamiltonian:

$$
H-\mu N=\sum_{\vec{k} \sigma} \Psi_{\vec{k} \sigma}^{\dagger} \Psi_{\vec{k} \sigma} \epsilon_{k}+J \vec{S}_{\mathrm{imp}} \cdot \vec{S}_{\mathrm{el}}(r=0) .
$$

Here $\vec{S}_{\mathrm{imp}}$ is the impurity spin operator and $\vec{S}_{\mathrm{el}}$ is the electron spin density at position $\vec{r}$ :

$$
\vec{S}_{\mathrm{el}}(\vec{r}) \equiv \Psi_{\alpha}^{\dagger} \frac{\vec{\sigma}_{\alpha \beta}}{2} \Psi_{\beta}(\vec{r})
$$

where $\Psi_{\alpha}(\vec{r})$ annihilates a conduction electron of spin $\alpha$ at $\vec{r}$ and repeated spin indices are summed over. We will focus on the case $S_{\mathrm{imp}}=1 / 2$ although much of the discussion here carries over to higher spin. The dispersion relation will often be assumed to be that of free electrons:

$$
\epsilon_{k}=\frac{k^{2}}{2 m}-\epsilon_{F}
$$

although that is not crucial. (For a general review of the Kondo model, see Ref. [1].) Essentially the same model can be used to describe a quantum dot in the Coulomb blockade regime in a spin $1 / 2$ state, interacting with one or more metallic leads (which can be formed by a 2 dimensional electron gas or by quantum wires in a semi-conductor inversion layer).

The dimensionless parameter which measures the strength of the Kondo interaction is $J \nu \equiv \lambda_{0}$ where $\nu$ is the density of states at the Fermi energy. For the free electron dispersion in D dimensions, this is:

$$
\nu_{D}=k_{F}^{D-1} /\left(c_{D} v_{F}\right)
$$

where $c_{3}=2 \pi^{2}, c_{2}=2 \pi$ and $c_{1}=\pi$. Typically $J \nu \ll 1$ suggesting that perturbation theory could be useful. However, perturbation theory encouters infrared divergences at low energy scales. In general, an $n^{\text {th }}$ order term in perturbation theory for some physical quantity characterized by an energy $E$ is proportional to $[J \nu \ln (\mathcal{D} / E)]^{n}$, where $\mathcal{D}$ is an ultra-violet scale of order the bandwidth or Fermi energy. It is found that the leading logarithmic divergences can be summed by expressing perturbation theory in terms of the renormalized coupling constant at scale $E$ :

$$
\lambda(E) \approx \lambda_{0}+\lambda_{0}^{2} \ln (\mathcal{D} / E)+\ldots
$$

Note that $\lambda(E)$ increases in magnitude, as $E$ is lowered, assuming it is initially positive (antiferromagnetic). $\lambda(E)$ obeys the renormalization group equation:

$$
\frac{d \lambda}{d \ln E}=\beta(\lambda)=-\lambda(E)^{2}+(1 / 2) \lambda(E)^{3}+\ldots
$$

where $\beta(\lambda)$ is the $\beta$-function. Keeping only the quadratic term in this RG equation, we find:

$$
\lambda(E) \approx \frac{\lambda_{0}}{1-\lambda_{0} \ln (\mathcal{D} / E)}
$$


Note that $\lambda_{0}$, the bare coupling, is the value of the renormalized coupling at the ultraviolet scale, $\mathcal{D}$. As the energy scale is lowered, $\lambda$ becomes of $\mathrm{O}(1)$ at the Kondo energy scale:

$$
k_{B} T_{K}=\mathcal{D} e^{-1 / \lambda_{0}} .
$$

This renormalization group equation, (6) is derived by integrating out high energy Fourier modes, reducing the effective bandwidth from $O(D)$, in energy units, to $O(E)$. Once the bandwidth becomes narrow (as happens at low energies) the width in wave-vector is related to the width in energy by $E=v_{F} \hbar\left|k-k_{F}\right|$ where $v_{F}$ is the Fermi velocity. Thus we may equally well describe $\lambda(E)$ as the effective coupling at wave-vector scale $E /\left(\hbar v_{F}\right)$ as at energy $E$. From this perspective, $k_{B} T_{K} /\left(\hbar v_{F}\right)$ is the characteristic wave-vector scale at which the effective Kondo coupling becomes large. Thus it is natural to introduce the Kondo length scale:

$$
\xi_{K} \equiv \hbar v_{F} /\left(k_{B} T_{K}\right)
$$

(We henceforth set $k_{B}$ and $\hbar$ to one.) It seems appropriate to think of the effective Kondo coupling as growing at large distances, becoming large at length scales of order $\xi_{K}$. Equivalently, we might expect physical quantities depending on a length scale $r$ to be scaling functions of the ratio $r / \xi_{K}$ rather than depending on $r$ and $\xi_{K}$ separately.

There is an interesting analogy here with Quantum Chromodynamics (QCD) the theory of the strong interactions in high energy physics. The renormalization group equation for the effective QCD coupling, $g_{Q C D}$, (describing the interactions of gluons with themselves and with quarks) is the same as Eq. (6) at quadratic order. It also gets large as the energy scale is lowered, and small as it is raised. In this case, one switches back and forth from energy units to momentum units using the velocity of light, $c$. The characteristic energy scale, $\Lambda_{Q C D}$ where $g_{Q C D}$ becomes $O(1)$ is of order $1 \mathrm{GeV}$, the mass of the proton, and the corresponding length scale is or order the Compton wavelength of the proton, 1 Fermi or $10^{-15} \mathrm{~m}$. If the quarks inside a proton are probed with high energy (short wavelength) photons they appear nearly free. On the other hand, they exhibit confinement (with an interaction growing linearly with separation) at long distances. In high energy physics it is commonplace to go back and forth freely between energy and length units using $c$ (and $\hbar$ ). However, there are well understood pitfalls in taking this picture of the effective coupling getting weak at high energies and strong at low energies, too literally. (These pitfalls occur both in the energy and distance picture, and do not seem to be particularly related to the distance viewpoint.) In this review article, we will be concerned with the validity of the corresponding picture for the Kondo model.

The $\delta$-function form of the Kondo interaction implies that only the s-wave harmonic interacts with the impurity so that the model becomes fundamentally 1-dimensional. After linearizing the dispersion relation about the Fermi surface, the low energy effective Hamiltonian becomes:

$$
H=\frac{i v_{F}}{2 \pi} \int_{0}^{\infty} d r\left[\psi_{L}^{\dagger} \frac{d}{d r} \psi_{L}-\psi_{R}^{\dagger} \frac{d}{d r} \psi_{R}\right]+v_{F} \lambda \vec{S}_{\mathrm{imp}} \cdot \vec{S}_{\mathrm{el}}(0) .
$$

Here $r$ is the radial coordinate and $\psi_{L / R}$ represent incoming and outgoing waves, with the boundary condition:

$$
\psi_{L}(0)=\psi_{R}(0) .
$$

These are defined in terms of the s-wave part of the 3D electron annihilation operator, $\Psi(\vec{r})$ by:

$$
\Psi(\vec{r})=\frac{1}{\sqrt{2} \pi r}\left[e^{-i k_{F} r} \psi_{L}(r)-e^{i k_{F} r} \psi_{R}(r)\right]+\ldots
$$

where the ... represents higher spherical harmonics. Note that we have normalized the fermion fields as in Ref. [2] so that:

$$
\left\{\psi_{L}^{\dagger}(r), \psi_{L}\left(r^{\prime}\right)\right\}=2 \pi \delta\left(r-r^{\prime}\right) .
$$

In the limit of zero Kondo interactions, $\lambda_{0}=0$, this describes a relativistic Dirac fermion with the Fermi velocity playing the role of the velocity of light. In such a model it is natural to go back and forth between energy and length units using $v_{F}$. Note that it is crucial to this estimate of $\xi_{K}$ that only one channel (the s-wave) couples to the Kondo impurity, allowing a mapping into a 1D model. While this happens in a variety of circumstances, including the case of a quantum dot coupled to 2DEG's, there are also important cases where it can fail, which will be discussed later.

An intuitive picture of this Kondo length scale can be obtained from considering the low energy strong coupling behavior of the model. This is most easily understood from a 1 dimensional tight-binding version of the model with Hamiltonian:

$$
H=-t \sum_{j=0}^{\infty}\left(c_{j}^{\dagger} c_{j+1}+h . c .\right)+J S_{\mathrm{imp}} \cdot \vec{S}_{\mathrm{el}}(0)
$$


The strong coupling limit is easily understood. When $J \gg t>0$, one electron gets trapped at the origin to form a singlet with the impurity spin. Note that this "Kondo screening" actually corresponds to the formation of an entangled state between the impurity spin and one conduction electron. The other electrons can do whatever they want except that they cannot enter or leave the origin since this would break up the Kondo singlet, costing an energy of $O(J)$. They effectively feel an infinite repulsion from the origin, corresponding to a $\pi / 2$ phase shift. This is simply a boundary condition on otherwise free electrons.

While the strong coupling limit is trivial, we are actually interested in the case of weak bare coupling, at low energies and long distances, where the effective coupling becomes strong. It is known from various approximate and exact calculations that the low energy physics (at $E \ll T_{K}$ ) is described by a local spin singlet state and noninteracting electrons with a $\pi / 2$ phase shift. On the other hand, the physics at intermediate energy scales, of $O\left(T_{K}\right)$ is complicated. To form a spin singlet with an $\mathrm{S}=1 / 2$ impurity, only one electron is needed and an intuitive picture is that one electron is "removed from the Fermi sea" for this purpose. However, unlike the simple case of large bare coupling, it would be quite wrong to think that this electron is localized at the origin. The natural length scale over which we may think of this electron's wave-function being non-negligible is $\xi_{K}$. Such a naive picture must be used with caution. At best, it is valid only at long distances and low energies. If we probe the screened impurity with a long wavelength probe, this picture may apply. At shorter distances, it certainly breaks down. While this is only an intuitive picture, it nonetheless seems reasonable that $\xi_{K}$ will appear as a characteristic scale in any distance dependent physical property of a Kondo system. The nature of the crossover at $\xi_{K}$ is the subject of this review.

Nozières' local Fermi liquid theory is well-known to provide a powerful way of studying the behaviour of distanceindependent quantities at low $T \ll T_{K}$. It turns out to also be useful for studying distance-dependent quantities at large distances $r \gg \xi_{K}$. In this approach, the screened impurity spin is eliminated from the effective Hamiltonian which, in lowest approximation, just consists of non-interacting electrons with a $\pi / 2$ phase shift. Then, interactions at the origin are added for these phase shifted electrons, which originate from virtual excitations of the screened impurity complex. We may simply list all possible interactions at the origin allowed by symmetry. Assuming exact particle-hole symmetry, there is only one important leading irrelevant interaction:

$$
H_{\text {int }}=-\frac{v_{F}^{2}}{6 T_{K}} \vec{J}_{L}^{2}(0)
$$

Here the current operator is defined as:

$$
\vec{J}_{L}(r) \equiv \psi_{L}^{\dagger}(r) \frac{\vec{\sigma}}{2} \psi_{L}(r)
$$

$H_{\text {int }}$ contains a dimension 2 operator, so its coupling constant must have dimensions of inverse energy. On general grounds, we expect that this coupling constant will be of order the crossover scale, $1 / T_{K}$. The factor of $1 / 6$ in Eq. (15) is just a matter of convenience. With this normalization, a calculation of the impurity susceptibility, to first order in this interaction gives:

$$
\chi(T) \rightarrow \frac{1}{4 T_{K}} .
$$

[This factor of $1 / 4$ in $\chi(T)$ is conventional since in the high $T \gg T_{K}$ limit we obtain the free impurity result, $\chi(T) \rightarrow 1 /(4 T)$.] We emphasize that various other definitions of $T_{K}$ are in common use, differing by factors of $\mathrm{O}(1)$.

Perhaps surprisingly, distance dependent aspects of Kondo physics are much less well-studied than energy dependent ones. At a theoretical level this may be a consequence of the fact that it is very difficult to get distance dependence from two of the most powerful methods of studying the Kondo effect: the Numerical Renormalization Group (NRG) method, introduced by Wilson, $\frac{3}{\underline{3}}$ and the Bethe ansatz (BA) solution, discovered by Andrei $\underline{\underline{4}}$ and Weigmann $\underline{\underline{5}}$. The difficulty with NRG lies in the "logarithmic discretization" or the fact that an effective 1D tight binding model is introduced in which the hopping parameter decays exponentially with distance from the impurity. This is, in fact, only an approximation to the full problem and one which seems to fail to keep track of length-dependence properly. However, more recently a way around this difficulty is being exploited $\frac{6.7}{}$ which involves a more complicated and numerically costly version of NRG. The difficulty with BA seems to be that while exact wave-functions are calculated, they are given in such a complicated form that it is very challenging to calculate any Green's functions with them. Thus much of our understanding of length dependence comes from perturbation theory and analytic RG arguments, various mean field theories, exact diagonalization of short systems and Density Matrix Renormalization Group (DMRG) numerical results which are also restricted to fairly short systems (up to 32 sites). The reasons why this exponentially large scale, $\xi_{K}=v_{F} / T_{K}$, has never been seen experimentally are probably that the associated crossover effects at this length scale are rather weak and subtle and that the basic Kondo model may not be adequate to describe the experimental systems used. Some inprovements to the model that might be neccessary are: including 
charge fluctuations (as in the Anderson model) taking into account a finite density of magnetic impurities, taking into account a finite density of non-magnetic impurities and taking into account electron-electron interactions even away from the impurity location. Thus searches for effects at this length scale represent both an experimental challenge to find sufficiently ideal systems and also an opportunity to study the limits of validity of the basic model.

We will be concerned with two types of length dependence. The first type, analysed in Sec. II involves a single impurity in an infinite host, described by the Hamiltonian of Eq. (11). We consider various observables as a function of distance from the impurity. The first one (Sub-Section (IA) is the Knight shift, measurable in nuclear magnetic resonance (NMR) experiments. This is simply the magnetic polarization of the electrons as a function of distance from the impurity, in linear response to a magnetic field (applied to both the impurity and the conduction electrons). The second (Sub-Section 【B is the charge density (Friedel) oscillations, as a function of distance from the impurity. These could be observed by scanning tunneling microscopy (STM) for a magnetic impurity on a metallic surface. The third (Subsection IIC) is the ground state equal time correlation function of the impurity spin and electron spin density at distance $r$ from the impurity. While probably not experimentally observable, this has a rather direct interpretation in terms of measuring the spatial probability distribution for the electron forming the spin singlet with the impurity. The second type of length dependence, analysed in Sec. III, occurs in mesocopic samples containing a single localized $\mathrm{S}=1 / 2$ where the size of some part of the device is comparable to $\xi_{K}$. Here we consider four different situations. Two of them involve a $\mathrm{S}=1 / 2$ quantum dot coupled to a finite length one-dimensional quantum wire. In the first case, (Subsection III A) this wire is closed into a ring and we consider the persistent current through it as a function of the ring length and magnetic flux. In the second case, (Subsection IIIB) the ring is straight with the quantum dot coupled to one end and the other end open. In Subsection IIIC we discuss an $\mathrm{S}=1 / 2$ quantum dot embedded in the centre of a finite length quantum wire which is tunnel-coupled to leads. In Subsection IIID, we contrast these strictly 1D models with the case of a magnetic impurity inside a three dimensional magnetic sample containing non-magnetic disorder: the Kondo box model. $\underline{8}$ Given certain simplifying assumptions about the disorder, the characteristic Kondo length scale is much smaller than $v_{F} / T_{K}$. It turns out that this is not just a consequence of the 3 dimensionality but also of the disorder. In an ideal 3D Kondo box $\xi_{K}$ would again be the characteristic length, as we discuss. Electron-electron interactions away from the impurity are ignored in all sections except IIIB, a quantum dot at the end of a finite quantum wire, where they are included using Luttinger liquid techniques. Sec. IV] contains conclusions.

\section{IMPURITY IN AN INFINITE HOST}

In this section, we consider the Hamiltonian of Eq. (10) for a single impurity interacting with electrons in an infinite volume. This is the traditional model for magnetic impurities in a metal. Note, however, that we ignore the presence of other impurities. A simplifying feature of this situation is that the mapping onto a single channel 1D system is exact. As we will see in sub-section IIID this is not generally the case when we put a 3D system into a finite box. Reflections off the boundary of the "box" greatly complicate the model.

An immediate question that arises is: how dilute must the magnetic impurities be to justify a single impurity approximation? Given the screening cloud picture, one might come to the pessimistic conclusion that the average separation of impurities should be much larger than $\xi_{K}$ :

$$
n_{\operatorname{imp}}^{-1 / 3} \gg \xi_{K} ? ?
$$

(Here $n_{\text {imp }}$ is the impurity density.) Such low densities are rarely, perhaps never, acheived in experiments on metals with dilute concentrations of magnetic impurities. Since typical ratios of $\xi_{K}$ to lattice constant can be in the thousands this would require densities $n_{\text {imp }} \ll 10^{-9}$ per unit cell. The reason ${ }^{9}$ that the condition is much weaker than this is basically that screening cloud wave-functions around different impurities become nearly orthogonal even when their centres are much closer together than their size. To see this consider two spherically symmetric wave-functions, centred around the points $\pm \vec{R} / 2$ but otherwise identical, only containing Fourier modes within a narrow band of wave-vectors of width $\xi_{K}^{-1}$ around $k_{F}$. The overlap is:

$$
O(R)=\int d^{3} r \psi^{*}(|\vec{r}+\vec{R} / 2|) \psi(|\vec{r}-\vec{R} / 2|) .
$$

In terms of the Fourier transform of the screening cloud wave-function, this becomes:

$$
O(R)=\int \frac{d^{3} k}{(2 \pi)^{3}} e^{i \vec{k} \cdot \vec{R}}|\psi(k)|^{2}=\int_{0}^{\infty} d k k^{2}|\psi(k)|^{2} \frac{\sin (k R)}{2 \pi^{2} k R} .
$$


We expect the screening cloud wave-function to decay on the length scale $\xi_{K}$ but to oscillate at the Fermi wave-vector. It should be built out of wave-vectors within $\xi_{K}^{-1}$ of $k_{F}$. Thus we may assume:

$$
|\psi(k)|^{2} \approx\left(\xi_{K} / k_{F}^{2}\right) f\left[\left(k-k_{F}\right) \xi_{K}\right]
$$

where the scaling function $f(y)$ obeys the normalization conditon:

$$
\int d y f(y)=2 \pi^{2}
$$

in order that $O(0)=1$. Thus,

$$
O(R)=\left(1 / 2 \pi^{2}\right) \int d y f(y) \sin \left[k_{F} R+\left(R / \xi_{K}\right) y\right] / k_{F} R .
$$

For $R \ll \xi_{K}$, this reduces to:

$$
O(R)=\sin \left(k_{F} R\right) / k_{F} R
$$

independent of the details of the wave-function. Thus screening clouds centred on different impurities have negigible overlap provided that they are separated by a distance $R \gg k_{F}^{-1}$. A simple estimate of the condition for validity of the single impurity Kondo model is provided by the Nozìres exhaustion principle. There must be enough available conduction electron states, within $\xi_{K}^{-1}$ of the Fermi wave-vector, to form linearly independent screening wave-functions around each impurity. This gives the conditions on the average impurity separation:

$$
\begin{aligned}
n_{i m p}^{-1 / 3} & \gg \xi_{K}^{1 / 3} k_{F}^{-2 / 3}(3 D) \\
n_{i m p}^{-1 / 2} & \gg \xi_{K}^{1 / 2} k_{F}^{-1 / 2}(2 D) \\
n_{i m p}^{-1} & \gg \xi_{K}(1 D) .
\end{aligned}
$$

\section{A. Knight Shift}

The Knight shift is proportional to the $r$-dependent polarization of the electron spin density in linear response to an applied magnetic field. In NMR experiments, the total effective magnetic field felt by a nucleus is measured, from the nuclear resonance frequency. In addition to the applied magnetic field there is an additional contribution arising from the hyperfine interaction between the nuclear spin and the surrounding electron spins. Assuming for simplicity that this hyperfine interaction is very short range, the Knight-shift is just proportional to the local spin density at the location of the nucleus. For sufficiently weak applied fields, this is $\propto \chi(r)$, the local susceptibility. In the absence, of the magnetic impurity, this is simply the $r$-independent Pauli susceptibility, $\chi_{0}$. The Kondo interaction leads to an additional $r$-dependent term, which vanishes far from the impurity. Let

$$
\vec{S}_{\mathrm{el}} \equiv \int d^{3} r S_{\mathrm{el}}(\vec{r})
$$

be the total electron spin operator. The total conserved spin operator is:

$$
\vec{S}_{\mathrm{tot}} \equiv \vec{S}_{\mathrm{imp}}+\vec{S}_{\mathrm{el}}
$$

The $r$-dependent magnetic susceptibility is:

$$
\chi(r) \equiv<S_{\text {el }}^{z}(\vec{r}) S_{\text {tot }}^{z}>/ T .
$$

[We ignore the possible difference of $g$-factors for impurity and conduction electrons for simplicity; this is discussed in Ref. [10,11].] $\chi(r)$ contains a constant term, present at zero Kondo coupling, which is simply the usual Pauli susceptibility, $\nu / 2$. Since only the s-wave harmonic couples to the impurity spin, the other terms come entirely from the s-wave component of the electron field. It is convenient to take advantange of the boundary condition of Eq. (11), to make an "unfolding transformation", regarding $\psi_{R}(r)$ as the continuation of $\psi_{L}(r)$ to the negative $r$-axis:

$$
\psi_{R}(r)=\psi_{L}(-r)
$$


The s-wave part of the spin density operator may then be written:

$$
\vec{S}_{\mathrm{el}}^{\mathrm{S}-\mathrm{wave}}(\vec{r}) \approx \frac{1}{8 \pi r^{2}}\left[\vec{J}_{L}(r)+\vec{J}_{L}(-r)+e^{2 i k_{F} r} \psi_{L}^{\dagger}(r) \frac{\vec{\sigma}}{2} \psi_{L}(-r)++e^{-2 i k_{F} r} \psi_{L}^{\dagger}(-r) \frac{\vec{\sigma}}{2} \psi_{L}(r)\right]
$$

We expect this the continuum limit approximation to be valid at sufficiently long distances, $r \gg k_{F}^{-1}$. Thus, we see that the local susceptibility takes the form $\frac{13}{3}$ at $r \gg k_{F}^{-1}$ :

$$
\chi(r)-\nu / 2 \rightarrow \frac{1}{8 \pi^{2} r^{2} v_{F}}\left[\chi_{\mathrm{un}}(r)+\chi_{2 k_{F}}(r) 2 \cos 2 k_{F} r\right]
$$

where the uniform and $2 k_{F}$ parts are slowly varying functions of $r$. These can be written in the 1D field theory as:

$$
\begin{aligned}
\chi_{\mathrm{un}}(r) & \equiv v_{F} \int_{0}^{\beta} d \tau<\left[\psi_{L}^{\dagger}(r, \tau) \frac{\sigma^{z}}{2} \psi_{L}(r, \tau)+\psi_{L}^{\dagger}(-r, \tau) \frac{\sigma^{z}}{2} \psi_{L}(-r, \tau)\right] S_{\mathrm{tot}}^{z}(0)> \\
\chi_{2 k_{F}}(r) & \equiv-v_{F} \int_{0}^{\beta} d \tau<\psi_{L}^{\dagger}(r, \tau) \frac{\sigma^{z}}{2} \psi_{L}(-r, \tau) S_{\mathrm{tot}}^{z}(0)>
\end{aligned}
$$

Here $\vec{S}_{\text {tot }}$ is defined as in Eq. (29) but we may restrict the electron part to its s-wave harmonic only, since the other harmonics give zero contribution to the Green's functions of Eq. (34):

$$
\vec{S}_{\mathrm{el}}=\frac{1}{2 \pi} \int_{-\infty}^{\infty} d r \psi_{L}^{\dagger}(r) \frac{\vec{\sigma}}{2} \psi_{L}(r)+\ldots
$$

It can be proved, $, 10,11$ to all orders in perturbation theory, that

$$
\chi u n(r)=0 .
$$

In the 1D field theory, $\chi_{2 k_{F}}(r)$ obeys a simple renormalization group equation with zero anomalous dimension which expresses how physical quantities change under a change of ultraviolet cut off and of bare coupling:

$$
\left[\mathcal{D} \frac{\partial}{\partial \mathcal{D}}+\beta(\lambda) \frac{\partial}{\partial \lambda}\right] \chi_{2 k_{F}}\left(T, \lambda, \mathcal{D}, r T / v_{F}\right)=0 .
$$

This simple form follows because only operators defined at $r=0$ have anomalous dimensions in a boundary field theory. Thus there is no anomalous dimension for $\psi_{L}^{\dagger}(r) \psi_{L}(-r)$. Furthermore, $\vec{S}_{\text {tot }}$ has zero anomalous dimension because it is conserved, commuting with the full Hamiltonian. This RG equation implies that $\chi_{2 k_{F}}\left(T, \lambda_{0}, D, r T / v_{F}\right)$ which is, a priori a dimensionless function of 3 dimensionless variables, $\lambda_{0}$, (the bare coupling), $\mathcal{D} / T$ and $r T / v_{F}$, can be written as a function of the renormalized coupling at scale $T, \lambda(T)$ and $r T / v_{F}$ only. i.e. both the bare coupling, $\lambda_{0}$ and the ultraviolet cut-off, $D$ can be eliminated in favour of a single variable, $\lambda(T)$. This is a basic consequence of renormalizability. An equivalent statement is that we may express $\chi_{2 k_{F}}$ as a function of the ratio $r T / v_{F}$ and $\lambda(r)$, the effective coupling at energy scale $v_{F} / r$. This follows from the RG equation, Eq. (6) which implies:

$$
F[\lambda(r)]-F[\lambda(T)]=\ln \left(v_{F} / r T\right)
$$

where $F(\lambda)$ is the indefinite integral of $1 / \beta(\lambda)$ :

$$
F(\lambda) \equiv \int^{\lambda} \frac{1}{\beta\left(\lambda^{\prime}\right)} d \lambda^{\prime} .
$$

Eq. (38) can be solved to express $\lambda(T)$ in terms of $\lambda(r)$ and a function of $r T / v_{F}$.

Results on $\chi_{2 k_{F}}(r)$ were presented $\underline{10}-\underline{12}$ to third order in pertubation theory in the Kondo coupling, $\lambda_{0}$, at finite $T$. In the $T \rightarrow 0$ limit these become:

$$
\chi_{2 k_{F}}=\frac{\pi v_{F}}{8 r T}\left[\lambda_{0}+\lambda_{0}^{2} \ln (\tilde{\Lambda} r)+\lambda_{0}^{3} \ln ^{2}(\tilde{\Lambda} r)+.5 \lambda_{0}^{3} \ln (\tilde{\Lambda} r)-\lambda_{0}^{3} \ln (\mathcal{D} / T)+\text { constant } \cdot \lambda_{0}^{3}\right] .
$$

Here $\tilde{\Lambda} \equiv 4 \pi D / v_{F}$. The presence of the $\lambda_{0}^{3} \ln (D / T)$ term is very important. If this term were absent, we could safely take $T \rightarrow 0$ without obtaining any infrared divergence in perturbation theory, at sufficiently small $r$. In that case, it 
would be convenient to express $\chi_{2 k_{F}}$ in terms of $\lambda(r)$ only. At small $\lambda(r)$ perturbation theory would apparently be valid. However, the presence of this term implies that this doesn't work. We can write instead:

$$
\chi_{2 k_{F}} \approx \pi \frac{v_{F}}{8 r T}\left[\lambda(r)+\text { constant } \cdot \lambda^{3}(r)\right][1-\lambda(T)]
$$

An important lesson from this expression is that the behaviour of the local susceptibity does not become perturbative, at low $T$, even at small $r$. This contradicts the naive expectation from the analogy with QCD, discussed in Scc. I. Nonetheless, it does suggest that there may be some crossover, or change in behaviour as we go from the region $r \ll \xi_{K}$ where $\lambda(r) \ll 1$ to $r \gg \xi_{K}$ where $\lambda(r)$ is large. A conjecture was made in Ref. [11] for the behaviour at all $r \ll v_{F} / T:$

$$
\chi_{2 k_{F}}(r) \rightarrow \frac{\pi v_{F}}{r} f\left(r / \xi_{K}\right) \chi(T)
$$

where $\chi(T)$ is the total, $r$-independent, impurity susceptility; i.e. the linear response of the total magnetization to an applied field, with the bulk Pauli term subtracted off. Here $f$ is some scaling function, depending on $r / \xi_{K}$. This conjecture is consistent with both the perturbative results given above, Fermi liquid results at long distances and low $T, r \gg \xi_{K}, T \ll T_{K}$, and also with results on the $k$-channel Kondo model in the large $k$ limit. $\chi(T)$ is well-known from a variety of methods, including BA. At $T \gg T_{K}$ it has the pertubative behaviour:

$$
\chi(T) \rightarrow \frac{1-\lambda(T)}{4 T} \approx \frac{1}{4 T}\left[1-\frac{1}{\ln \left(T / T_{K}\right)}\right]
$$

At low $T \ll T_{K}, \chi(T) \rightarrow 1 /\left(4 T_{K}\right)$. For intermediate $T$ it is quite well approximated by $\chi(T) \approx .17 /\left[\left(T+.65 T_{K}\right)\right]$. We only know the form of the other scaling function, $f\left(r / \xi_{K}\right)$ asymptotically, at short and long distances. At short distances, $r \ll \xi_{K}$ it is perturbative:

$$
f(r) \rightarrow \lambda(r) \approx \frac{1}{\ln \left(\xi_{K} / r\right)}
$$

In the opposite limit, $r \gg \xi_{K}$ we may also determine $13 f(r)$ from Fermi liquid theory. In the limit of low $T \ll T_{K}$ and large $r \gg \xi_{K}$ first order perturbation theory in the Fermi liquid interaction of Eq. (15) gives the proposed form, eq. (42) with:

$$
f\left(r / \xi_{K}\right) \rightarrow 4 r /\left(\pi \xi_{K}\right)+1 / 3+O\left(\xi_{K} / r\right)
$$

At intermediate length scales, of $O\left(\xi_{K}\right)$, the function $f\left(r / \xi_{K}\right)$ must somehow crossover between $1 / \ln \left(r / \xi_{K}\right)$ and $4 r /\left(\pi \xi_{K}\right)$. Determining the function in this region requires numerical methods.

At $T \gg T_{K}$, we found that the infrared divergences of perturbation theory are cut-off and weak coupling behaviour ensues, at any distance. There is no crossover at $r \approx \xi_{K}$ in this case. Our result to third order in $\lambda$ is:

$$
\chi_{2 k_{F}}(r) \approx\left(3 \pi^{2} / 4\right) \lambda(T)^{2}[1-\lambda(T)] e^{-2 \pi r T / v_{F}} .
$$

Our proposed scaling form for the local susceptibility when $r \ll v_{F} / T$ :

$$
\chi(r)-\nu / 2 \rightarrow \frac{\cos 2 k_{F} r}{4 \pi r^{3}} f\left(r / \xi_{K}\right) \chi(T)
$$

makes it clear that NMR measurements of the Knight shift would be a very difficult way of detecting the Kondo screening cloud. Typically an NMR signal is only picked up for nuclei within a few times $k_{F}^{-1}$ of an impurity spin. Over this range, the $r$-dependence of Eq. (47) is dominated by the first factor, $\cos 2 k_{F} r / r^{3}$. The slowly varying factor in the envelope of the oscillations, $f\left(r / \xi_{K}\right)$, could not be measured unless signals could be picked up out to distances of $O\left(\xi_{K}\right)$. At such large distances, the Knight shift is very small due to the factor of $1 / r^{3}$. In fact, this form is qualitatively consistent with existing NMR experiments which looked for the Kondo screening cloud 14 An important further complication in the experiments is that a given nucleus will feel a contribution to its Knight shift from many magnetic impurities at various distances. These contributions are expected to be simply additive, provided that the impurities are sufficiently diulte; see discussion at the beginning of Sec. II. Nonetheless, it would be extremely difficult to disentangle these various contributions and require extremely accurate measurements of the Knight shift to determined the tiny contributions from distance impurities.

An important lesson here is that the Kondo screening cloud is difficult to detect because it is so large. To see it, one must look for a crossover at large distances but at these distances the effects are weak precisely because the distances 
are so large. The Kondo screening "cloud" is perhaps better described as a "faint fog". 15 It is worth noting that the situation is much better for a sample of reduced dimensionality. Our formula for the local susceptibility, Eq. (33), carries over directly to a $2 \mathrm{D}$ or $1 \mathrm{D}$ sample, with the factor of $1 / r^{2}$ simply becoming proportional to $1 / r^{D-1}$. In the 1D case, $\chi(r) / \cos \left(2 k_{F} r\right)$ crosses over from $1 /\left[r \ln \left(\xi_{K} / r\right)\right]$ for $r \ll \xi_{K}$ to a constant at $r \gg \xi_{K}$, actually growing with distance. However, the diluteness condition is also modified in $1 \mathrm{D}$ to

$$
n_{\mathrm{imp}}^{-1} \gg \xi_{K}
$$

as discussed at the beginning of Sec. [II.

\section{B. Density Oscillations}

While the Kondo effect is associated with the spin degrees of freedom of the conduction electrons, it also has an interesting effect on the charge density in the vicinity of the impurity ${ }^{7,16}$ This is perhaps a bit surprising, in light of spin-charge separation in 1D. It turns out that density oscillations only occur when particle-hole (p-h) symmetry is broken. In particular, none occur in a half-filled tight-binding model, which has exact particle-hole symmetry. Importantly, the Kondo interaction itself preserves p-h symmetry.

These density oscillations turn out to be quite a bit simpler to analyse than the Knight shift, $\chi(r)$, discussed in sub-section IA This is because, at long distances, $r \gg k_{F}^{-1}$, they can be expressed as a half Fourier transform of a well-studied scaling function of $\omega / T_{K}$, the $\mathcal{T}$-matrix, $\mathcal{T}(\omega)$. Due to the $\delta$-function nature of the Kondo interaction, the exact retarded electron Green's function in the Kondo model can be written:

$$
-i \int_{0}^{\infty} d t e^{i \omega t}<\left\{\psi(\vec{r}, t), \psi^{\dagger}\left(\vec{r}^{\prime}, 0\right)\right\}>=G\left(\vec{r}, \vec{r}^{\prime}, \omega\right)=G_{0}\left(\vec{r}-\vec{r}^{\prime}, \omega\right)+G_{0}(\vec{r}, \omega) \mathcal{T}(\omega) G_{0}\left(-\vec{r}^{\prime}, \omega\right) .
$$

Here $G_{0}$ is the Green's function for the non-interacting case:

$$
G_{0}(r, \omega)=\int \frac{d^{3} k}{(2 \pi)^{3}} e^{i \vec{k} \cdot \vec{r}} \frac{1}{\omega-\epsilon_{k}+i \eta}
$$

The $\mathcal{T}$-matrix is used to determined the resistivity and thus plays an important role in the theory of the Kondo model. Although it is not accessible by BA, NRG techniques have been developed to calculate it, in addition to perturbative and Fermi liquid results and other approximations. The density is obtained from the Green's function in the standard way:

$$
\rho(r)=-\frac{2}{\pi} \int_{-\infty}^{0} d \omega \operatorname{Im} G(\vec{r}, \vec{r}, \omega) .
$$

Note that $G(\vec{r}, \vec{r}, \omega)$ itself has trivial $r$-dependence, determined entirely by $G_{0}(r)$. Only the frequency-dependence reflects the Kondo physics. However, once we integrate over $\omega$ in Eq. (51), this $\omega$-dependence introduces some interesting $r$-dependence. In the limit $r \gg k_{F}^{-1}$, we may use the asymptotic expression for $G_{0}(r)^{2}$. In D-dimensions, for the free-electron dispersion relation this is:

$$
G_{0}(r, \omega)^{2} \rightarrow-\frac{1}{v_{F}^{2}}\left(\frac{-i k_{F}}{2 \pi r}\right)^{D-1} \exp \left(2 i k_{F} r+2 i \omega r / v_{F}\right) .
$$

This asymptotic form of $G_{0}$ can be calculated for other dispersion relations and has a similar form. Thus we see that, at $r \gg k_{F}^{-1}$ the density oscillations induced by a Kondo impurity are given by the half-Fourier transform of the $\mathcal{T}$-matrix. Furthermore, for such large $r$, we expect this half Fourier transform to be dominated by frequencies $\leq v_{F} / r \ll v_{F} k_{F} \propto D$. In this frequency range, the $\mathcal{T}$-matrix is a universal scaling function of $\omega / T_{K}$.

It is important in calculating the density oscillations to take into account additional potential scattering, arising from the magnetic impurity, in addition to the Kondo interaction. This was ignored in sub-section I A since it is not very important for the Knight shift. This corresponds to an additional term in the Kondo Hamiltonian, Eq. (1) of the form:

$$
\delta H=V \sum_{\sigma} \psi_{\sigma}^{\dagger}(0) \psi_{\sigma}(0) .
$$

We refer to the s-wave phase shift at the Fermi surface due to the potential scattering as $\delta_{P}$. The $\mathcal{T}$-matrix, at $\omega \ll D$ can be expressed in terms of $\delta_{P}$, a universal function, $t_{K}\left(\omega / T_{K}\right)$ and the density of states in D-dimensions, $\nu_{D}$ :

$$
\mathcal{T}(\omega)=\left\{e^{2 i \delta_{P}}\left[t_{K}\left(\omega / T_{K}\right)+i\right]-i\right\} /\left(2 \pi \nu_{D}\right)
$$


Note that, in the particle-hole symmetric case, $\delta_{P}=0, \mathcal{T}=t_{K} /\left(2 \pi \nu_{D}\right)$. By rescaling the frequency integration variable, and using $v_{F} / T_{K}=\xi_{K}$, we see that the half-Fourier transform occuring in $\rho(r)$ may be expressed in terms of a universal scaling function, $F\left(r / \xi_{K}\right)$ :

$$
\int_{-\infty}^{0} d \omega e^{2 i \omega r / v_{F}} t_{K}\left(\omega / T_{K}\right) \equiv\left[v_{F} /(2 r)\right]\left[F\left(r T_{K} / v_{F}\right)-1\right] .
$$

Particle-hole symmetry of the model with no potential scattering (from which $t_{K}$ can be determined) implies that $t_{K}^{*}\left(\omega / T_{K}\right)=-t_{K}\left(-\omega / T_{K}\right)$. Furthermore, since $t_{K}$ is related to the retarded Green's function it is analytic in the upper half plane. These two conditions imply that $F\left(r / \xi_{K}\right)$ is real. On the other hand, note that it depends on both real and imaginary parts of $t_{K}$. This makes it challenging to determine the density oscillations numerically, since while reasonably accurate results may exist for $\operatorname{Im} t_{K}$, this is not the case for the real part. From Eqs. (51), (544) and (55) we obtain the formula for the density oscillations at long distances from the impurity, $r \gg k_{F}^{-1}$ :

$$
\rho(r)-\rho_{0} \rightarrow \frac{C_{D}}{r^{D}}\left[\cos \left(2 k_{F} r-\pi D / 2+2 \delta_{P}\right) F\left(r / \xi_{K}\right)-\cos \left(2 k_{F} r-\pi D / 2\right)\right]
$$

where the constant is $C_{3}=1 /\left(4 \pi^{2}\right), C_{2}=1 /\left(2 \pi^{2}\right)$ and $C_{1}=1 /(2 \pi)$. The formula applies in $\mathrm{D}=1,2$ or 3 , with a spherically symmetric dispersion relation. It also applies to the 1D tight-binding model in which case $r$ is restricted to integer values. Note that in this case, there is exact p-h symmetry at half-filling with no potential scattering, corresponding to $k_{F}=\pi / 2$ and $\delta_{P}=0$. In this case, the right hand side of Eq. (56) vanishes identically as expected. p-h symmetry breaking is necessary to get non-zero density oscillations and the potential scattering phase shift, $\delta_{P}$ plays an important role in them.

The universal Kondo $\mathcal{T}$-matrix, $t_{K}\left(\omega / T_{K}\right)$, can be calculated at higher frequencies, $\omega \gg T_{K}$ using perturbation theory in the Kondo interaction. It can be calculated at low frequencies using Fermi liquid theory. We find that these expansions can be simply inserted into Eq. (55) to obtain expansions for $F\left(r / \xi_{K}\right)$ and hence the density oscillations at short distances, $r \ll \xi_{K}$ and long distances, $r \gg \xi_{K}$. The weak coupling result is

$$
t_{K}(\omega)=-\left(3 i \pi^{2} / 8\right)\left[\lambda_{0}^{2}+2 \lambda_{0}^{3} \ln (\mathcal{D} / \omega)+\ldots\right] .
$$

This may be written:

$$
t_{K}\left(\omega / T_{K}\right) \rightarrow-\left(3 \pi^{2} i / 8\right) \lambda^{2}(|\omega|) \approx-3 \pi^{2} i /\left[8 \ln ^{2}\left(|\omega| / T_{K}\right)\right] .
$$

The $\mathcal{T}$-matrix vanishes slowly at high frequencies. Substituting this weak coupling expansion into the integral defining $F$, Eq. (55) and integrating term by term gives:

$$
F=1-\left(3 \pi^{2} / 8\right)\left[\lambda_{0}^{2}+2 \lambda_{0}^{3} \ln (\tilde{\Lambda} r)+\ldots\right]
$$

where $\tilde{\Lambda}$ is of order $v_{F} / D$. We recognize the weak couplig expansion of the effective coupling at scale $r$ :

$$
F\left(r / \xi_{K}\right) \rightarrow 1-\left(3 \pi^{2} / 8\right) \lambda^{2}(r) \approx 1-3 \pi^{2} /\left[8 \ln ^{2}\left(\xi_{K} / r\right)\right] .
$$

Note that in the weak coupling limit $F \rightarrow 1$ and then Eq. (56) reduces to the standard formula for the Friedel oscillations induced by a non-magnetic impurity which produces a phase shift at the Fermi surface of $\delta_{P}$ (in the s-wave channel only).

Our results indicate that weak coupling behaviour occurs for the density oscillations at short distances $r \ll \xi_{K}$ even at $T=0$. This is quite different than what we found for the Knight shift in Sec. IIA. In that case we found, in Eq. (40), that the infrared divergences of perturbation theory were not completely cut off at short distances. However our conjecture, Eq. (42), implied that the non-perturbative aspect simply led, at low $T \ll v_{F} / r$, to a factor of $\chi(T)$ in $\chi(r)$ which was otherwise linear in $\lambda(r)$, Eq. (44). The density oscillations therefore show simpler behaviour at short distances.

At low frequencies, $\omega \ll T_{K}$, the Kondo $\mathcal{T}$-matrix can be calculated from Fermi liquid theory:

$$
t_{K} \rightarrow-i\left[2+i \pi \omega /\left(2 T_{K}\right)-3 \pi^{2} \omega^{2} /\left(16 T_{K}^{2}\right)+\ldots\right] .
$$

Substituting into Eq. (55), gives:

$$
F\left(r / \xi_{K}\right) \rightarrow-1+\pi \xi_{K} /(4 r)-3 \pi^{2} \xi_{K}^{2} /\left(32 r^{2}\right)+\ldots
$$

At large distances, $r \gg \xi_{K}, F \rightarrow-1$. From Eq. (56), we see that this is equivalent to shifting $\delta_{P} \rightarrow \delta_{P}+\pi / 2$ while setting $F=1$, the non-interacting value. In this limit the Kondo scattering simply changes the phase shift by $\pi / 2$, 
the familiar result. Thus, while we obtain the standard Friedel oscillation formula at both short and long distances, there is a non-trivial and universal crossover, determined by $F\left(r / \xi_{K}\right)$, at intermediate distances.

The density oscillations were calculated using an improved NRG method in Ref. 7]. In this approach Wannier states are introduced both at the impurity location and at the point of interest, $r$, fixing the problem with spatial resolution in the usual NRG approach. Results are shown in Fig. (11). The oscillations can be parametrized, at $r \gg k_{F}^{-1}$, by the form of Eq. (56). The function $F\left(r / \xi_{K}\right)$ fits the asymptotic predictions at large and small arguments, crossing over between 1 and -1 as expected. In fact, it is fairly well fit, throughout the crossover by the simple "one spinon approximation" $\underline{16,17}$

$$
t_{K}\left(\omega / T_{K}\right) \approx-2 i /\left[1-i \pi /\left(2 T_{K}\right)\right]
$$

giving an approximate formula for $F$ :

$$
F(u) \approx 1+(8 u / \pi) e^{4 u / \pi} \operatorname{Ei}(-2 u / \pi),
$$

where $E_{i}(x)$ is the exponential-integral function.

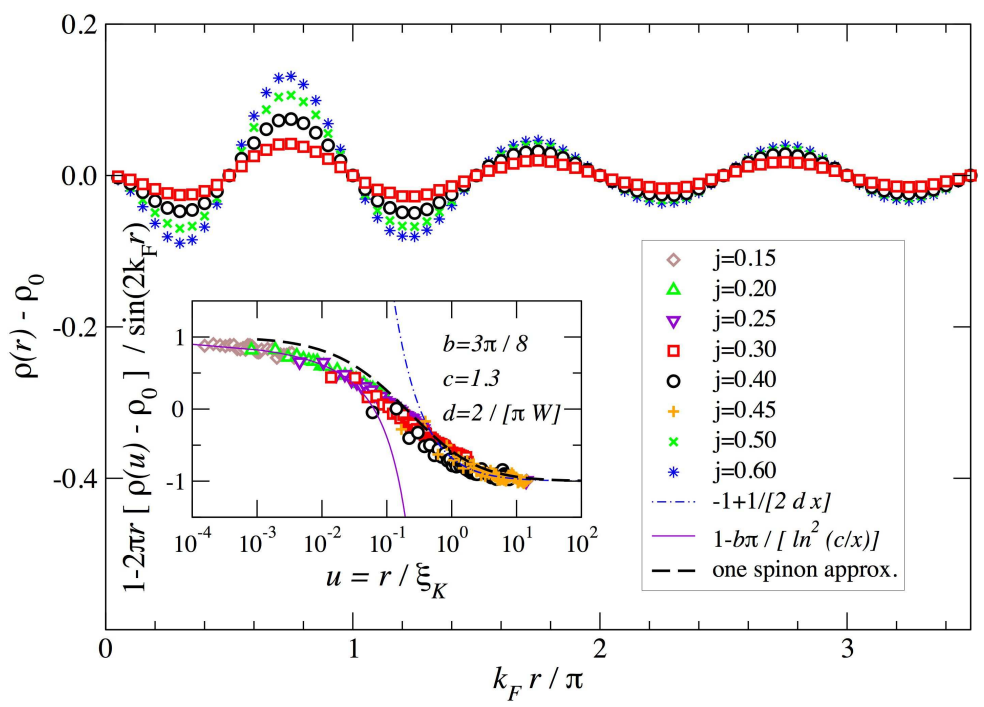

FIG. 1: NRG results on charge oscillations around a Kondo impurity coupled to 1D conduction electrons with particle-hole symmetry from Ref. 7]. Note that the oscillations vanish at $k_{F} r / \pi \in \mathbb{N}$. As shown in the inset, the properly rescaled envelope function of the oscillations (extracted as $\rho-\rho_{0}$ at the local maxima) for different Kondo couplings nicely collapse into one universal curve except for the points where $r \sim k_{F}^{-1}$. In the inset we show the analytical results for the asymptotics as well: Note the good agreement between the analytical results and the numerics.

Experimental measurement of the Kondo screening cloud via density oscillations would again be extremely challenging. The $1 / r^{D}$ factor in Eq. (56) implies that the oscillations would be extremely small at the length scale $\xi_{K}$. (Note that the number of oscillations that would need to be measured is of order $\xi_{K} k_{F} \gg 1$.) As for the Knight shift, the situation is improved in lower dimensions. The Kondo effect is apparently observed for magnetic impurities on metallic surfaces using Scanning Tunelling Microscopy (STM). In cases, where the Kondo interaction is predominantly with surface conduction electron states, Eq. (56) with $D=2$ should apply. The STM tunelling rate for tip energy $E$ is usually assumed to be proportional Eq. (51) but with the lower limit of integration replaced by $E$. This quantity could also be calculated from knowledge of $t_{K}$, but only when $E \gg T_{K}$ do we recover a scaling function of $r / \xi_{K}$ only.

\section{Impurity spin correlation function}

Another quantity which gives a very direct picture of the Kondo screening cloud is the equal time ground state correlator of the impurity spin with the spin density at a distance $r$ :

$$
\left.K(\vec{r}, T) \equiv<0\left|S_{\mathrm{el}}^{z}(\vec{r}, 0) S_{\mathrm{imp}}^{z}(0)\right| 0\right)>.
$$


Although this quantity was also discussed at finite $T$ in Ref. [10,11], we restrict our discussion here to the $T=0$ case. Unlike for the local susceptibility, no divergences are encountered in perturbation theory by taking $T \rightarrow 0$, at least to the (third) order studied. $K(r)$ can easily be seen to obey an exact sum rule. If we consider a finite system with an even number of electrons in total (including the impurity spin as an electron) then the ground state is a spin singlet:

$$
S_{\text {tot }}(0)^{z} \mid 0>=0
$$

Thus we have:

$$
0=<0\left|S_{\text {imp }}^{z}(0) S_{\text {tot }}(0)^{z}\right| 0>=1 / 4+\int d^{3} r K(\vec{r}) .
$$

A heuristic picture of $K(r)$ is obtained by writing:

$$
S_{\mathrm{el}}^{z}(\vec{r}) \approx s_{\mathrm{el}}^{z} \rho(\vec{r})
$$

Here $\rho(\vec{r})$ is the probability of the screening electron being at the location $\vec{r}$ and $s_{\text {el }}^{z}$ is a $\mathrm{S}=1 / 2$ operator, representing the spin of the screening electon, with $<S_{\text {imp }}^{z} s_{\text {el }}^{z}>=-1 / 4 . \rho(\vec{r})=|\phi(\vec{r})|^{2}$ where $\phi(\vec{r})$ is the screening cloud wavefunction. Then we obtain $K(r) \approx-\rho(r) / 4$ and the sum rule of Eq. (67) is simply the normalization condition on the screening electron's wave-function. As for the local susceptibility, discussed in sub-section IA at $r \gg k_{F}^{-1}$ we may decompose $K$ into uniform and $2 k_{F}$ parts:

$$
K(r) \rightarrow \frac{1}{8 \pi^{2} v_{F} r^{2}}\left[K_{\mathrm{un}}(r)+2 K_{2 k_{F}}(r)\right]
$$

where $K_{\mathrm{un}}$ and $K_{2 k_{F}}$ can be calculated in the 1D field theory. Perturbation theory in the Kondo coupling, valid at distances $r \ll \xi_{K}$, gives: 10,11

$$
\begin{aligned}
K_{\mathrm{un}} & \approx-\frac{\pi v_{F} \lambda(r)^{2}\left(1+\lambda_{0} / 2\right)}{2 r} \\
K_{2 k_{F}} & \approx \frac{\pi v_{F} \lambda(r)\left(1+\lambda_{0} / 2\right)}{8 r}
\end{aligned}
$$

Several differences are to be noted with the local susceptibility. First of all, we have obtained a finite result at $T=0$, as noted above. Secondly the uniform part is now non-zero. Thirdly, the result cannot be expressed in terms of the renormalized coupling at scale $r$ only, but also involves a factor containing the bare coupling, $\lambda_{0}$. This can be understood $^{10,11}$ from the fact that the impurity spin operator has a non-zero anomalous dimension:

$$
\gamma_{\mathrm{imp}} \approx \lambda^{2} / 2+\ldots
$$

However, this is not very important since this factor goes to 1 in the scaling limit of weak bare coupling. On the other hand, at $r \gg \xi_{K}$ we may calculate ${ }^{10,11,18} K(r)$ using Fermi liquid theory giving:

$$
K_{2 k_{F}} \rightarrow-(1 / 2) K_{\mathrm{un}} \rightarrow \text { const } \times \frac{\xi_{K} v_{F}}{r^{2}}
$$

Our heuristic interpretation then suggests a slow power-law decay for the the screening cloud electron probability density:

$$
\rho(r) \approx \text { const } \times \frac{\sin ^{2} k_{F} r \xi_{K}}{r^{4}}\left(r \gg \xi_{K}\right)
$$

On the other hand, at shorter distances, $r \ll \xi_{K}, K(r)$ is not negative definite and the heuristic picture of $K(r)$ is not valid.

\section{SPIN IN A MESOSCOPIC DEVICE}

Given the extreme difficulty of observing the Kondo screening cloud from length-dependent measurements in a macroscopic sample, discussed in Sec. II together with the fact that mesoscopic devices routinely contain components with dimensions of order 1 micron, it is natural to consider whether the best way of finally observing the Kondo 
screening cloud experimentally might be via size-dependent effects in such a device. We are interested in devices containing a quantum dot in the Coulomb blockade regime with a gate voltage tuned so that the number of electrons in the dot is odd, with a $S=1 / 2$ ground state. For simplicity, we will also assume that the tunelling from dot to leads is sufficiently weak that only virtual charge fluctuations of the dot need be considered so that the Kondo model is appropriate, rather than the Anderson model. Alternatively, we might consider one or more magnetic impurity atoms in a mesoscopic sample.

An important point is that, associated with a finite sample size, we have a finite spacing between energy levels. If the sample is 1 dimensional, then the level spacing will generally be $\Delta \approx v_{F} / L$ where $L$ is the sample size. Thus the condition $v_{F} / T_{K} \approx L$ is equivalent to $\Delta \approx T_{K}$. Thus, it sometimes argued that observing size dependence in this situation does not really show the existence of a Kondo screening cloud but "merely" that $T_{K}$ is the characteristic energy scale for the Kondo model. However, since as discussed in the previous sections, the Kondo screening cloud concept is really just the inevitable consequence of being able to convert an energy scale to a length scale using a factor of velocity, size effects can provide realizations of it. We may equally well say that the behaviour changes when the finite size gap becomes larger than $T_{K}$ or else when the Kondo screening cloud no longer fits inside the sample. If the sample should instead be regarded as 2 or 3 dimensional the finite size level spacing may be $\ll v_{F} / L$ depending on which energy levels are important and other details, discussed in Sec. IIID,

The Kondo model is used both to describe magnetic impurities in metals and also gated semi-conductor heterostructure quantum dots. The heterostructure (such as GaAs/AlGaAs) provides a fairly clean 2D electron gas (2DEG) buried some distance (often around 100 Angstroms) below the surface of the semi-conductor wafer. Gate voltages are applied to the surface to define point contacts and quantum dots. A quantum dot refers to an island of electrons, with the electron number (typically around 100 or less) controlled in unit steps by a gate voltage. When this number is odd, the quantum dot usually has an $\mathrm{S}=1 / 2$ ground state. The quantum dot can be connected by narrow point contacts to the left and right sides of the 2DEG. If the point contacts are close to being pinched off they only permit one channel of electrons to pass through, giving a $2 e^{2} / h$ conductance. In this case, a simplified model of the system involves effectively $1 \mathrm{D}$ leads. The quantum dot has a charging energy, $U$, as well as a gate voltage, such that the energy as a function of electron number, $N$, is $U\left(N-N_{0}\right)^{2}$ for a value of the parameter $N_{0}$ controlled by the gate. It is often convenient, theoretically, to use a 1D tight-binding model; universality of Kondo physics implies that such details are not important. The corresponding Hamiltonian is:

$$
H=H_{0}+H_{d}
$$

where

$$
H_{0}=-\left[t \sum_{j=-\infty}^{-2} c_{j \alpha}^{\dagger} c_{j+1, \alpha}-t \sum_{j=1}^{\infty} c_{j \alpha}^{\dagger} c_{j+1, \alpha}-t^{\prime} c_{-1 \alpha}^{\dagger} c_{0 \alpha}-t^{\prime} c_{0 \alpha}^{\dagger} c_{1 \alpha}+h . c .\right]
$$

and

$$
H_{d}=\epsilon_{0} n_{0}+U n_{0 \uparrow} n_{0 \downarrow} .
$$

Here $n_{0 \alpha}$ is the occupation number at site 0 for spin $\alpha$, with $n_{0} \equiv n_{0 \uparrow}+n_{0 \downarrow} \cdot t^{\prime}$ represents the tunelling amplitudes, through the left and right point contacts, to the leads. (We take these to be equal for simplicity but the generalization is straightforward.)

If the tunnelling amplitudes are sufficiently weak compared to $U$, then the quantum dot makes only virtual charge fluctuations and the system is in the Coulomb blockade regime. The conductance through the dot then usually begins to decrease as the temperature is lowered, if $N_{0}$ is close to an integer. In the case where $N_{0}$ is near an odd integer, so that the quantum dot behaves as an $\mathrm{S}=1 / 2$ impurity, we may make a Shrieffer-Wolff transformation to the corresponding Kondo model with Hamitonian:

$$
H=H_{0}+H_{K}
$$

where now:

$$
\begin{gathered}
H_{0}=-\left[t \sum_{j=-\infty}^{-2} c_{j \alpha}^{\dagger} c_{j+1, \alpha}-t \sum_{j=1}^{\infty} c_{j \alpha}^{\dagger} c_{j+1, \alpha}\right] \\
H_{K}=J\left(c_{-1}+c_{1}\right)^{\dagger} \frac{\sigma}{2}\left(c_{1}+c_{-1}\right) \cdot \vec{S}
\end{gathered}
$$


where $\vec{S}$ is the spin operator for the site 0 :

$$
S \equiv c_{0}^{\dagger} \frac{\vec{\sigma}}{2} c_{0}
$$

and the Kondo coupling is:

$$
J=2 t^{\prime 2}\left[\frac{1}{-\epsilon_{0}}+\frac{1}{U+\epsilon_{0}}\right]
$$

In general, a potential scattering term is also generated, of the same order of magnitude as $J$. To simplify the discussion, we focus here on the particle-hole symmetric case, $\epsilon_{0}=-U / 2$, where the potential scattering vanishes. We also assume that the system is at half-filling so that the particle-hole symmetry is exact. It is then easy to calculate the zero temperature conductance for this Kondo model in either limit, $J \ll t$ or $J \gg t$. The second limit is not physical for the underlying Anderson model of Eq. (74) but it is convenient to consider nonetheless as a low energy fixed point Hamiltonian. When $J=0$ the the Kondo Hamiltonian contains no terms linking left and right sides of the dot, so the conductance vanishes. When $J \gg t$, one electron gets trapped in the symmetric orbital, with annihilation operator:

$$
c_{s} \equiv\left(c_{1}+c_{-1}\right) / \sqrt{2}
$$

and forms a singlet with the impurity spin. At first sight, one might think that this would block the transport through the impurity. However, this is not so due to transport through the antisymmetric orbital with annihilation operator:

$$
c_{a} \equiv\left(c_{1}-c_{-1}\right) / \sqrt{2}
$$

Electrons entering from sites -2 or 2 can hop into this antisymmetric orbital without breaking the Kondo singlet. If instead they hop into the symmetric orbital there is a large energy cost of $O(J)$. At large $J / t$ we may obtain a low energy effective Hamiltonian from $H_{0}$ by projecting $c_{ \pm 1}$ onto $c_{a}$ :

$$
P c_{ \pm 1} P= \pm c_{a} / \sqrt{2}
$$

This gives:

$$
H_{\text {low }}=-t\left[\sum_{j=-\infty}^{-3} c_{j \alpha}^{\dagger} c_{j+1, \alpha}-\sum_{j=4}^{\infty} c_{j \alpha}^{\dagger} c_{j+1, \alpha}+\frac{1}{\sqrt{2}} c_{-2 \alpha}^{\dagger} c_{a \alpha}-\frac{1}{\sqrt{2}} c_{2 \alpha}^{\dagger} c_{a \alpha}+h . c .\right]
$$

The tranmission probability for this non-interacting electron problem is easily calculated: ${ }^{20}$

$$
T(k)=\sin ^{2} k \text {. }
$$

In particular, at half-filling, $k_{F}=\pi / 2$, the transmission probability at the Fermi energy is one (corresponding to a resonance). The conductance for this non-interacting model is given by the Landauer formula:

$$
G=\frac{2 e^{2}}{h} T\left(\epsilon_{F}\right)=\frac{2 e^{2}}{h} .
$$

Calculations show that, for a small bare Kondo coupling, the conductance grows with decreasing $T$, saturating at the perfect tranmission value, $2 e^{2} / h$, for $T \ll T_{K}$. These results can be obtained by expressing the conductance as a frequency integral of the imaginary part of the $\mathcal{T}$-matrix, introduced in sub-section II B at finite temperature, times the derivative of the Fermi function. Analytic formulas can be obtained for $G(T)$ in both the high $T$ and low $T$ limits using perturbation theory and Fermi liquid theory respectively. Calculations at intermediate $T$ are typically based on less controlled approximations. This increase of conductance with decreasing $T$, due to the Kondo effect, only sets in at low temperatures, following an initial decrease due to the onset of the Coulomb blockade.

Note that this behaviour is the inverse of what happens for a magnetic impurity in a bulk metal where it is the resistivity which grows with lowering $T$, not the conductance. The impurity behavior is more closely related to that for a side-coupled quantum dot where the conductance is $2 e^{2} / h$ for zero Kondo coupling (high $T$ ) and vanishes at low $T \ll T_{K}$. 


\section{A. Persistent current in a ring containing a Kondo impurity}

A calculationally simple situation in which to observe finite size effects is to close the leads embedding the quantum dot into a ring. The Hamiltonian is that of Eqs. (77) but the leads are now of finite length with periodic boundary conditions:

$$
\begin{aligned}
H_{0} & =-t \sum_{j=1}^{L-2}\left(c_{j \alpha}^{\dagger} c_{j+1, \alpha}+h . c .\right) \\
H_{K} & =J\left(c_{L-1}+c_{1}\right)^{\dagger} \frac{\vec{\sigma}}{2}\left(c_{L-1}+c_{1}\right) \cdot \vec{S} .
\end{aligned}
$$

While the conductance is now not measurable, one can instead calculate the persistent current in response to an enclosed magnetic flux, $\Phi=(\hbar c / e) \alpha$. The current, at $T=0$, is given by the derivative of the ground state energy with respect to the flux:

$$
j=-(e / \hbar) d E_{0} / d \alpha
$$

A perturbative calculation gives: 19,20

$$
\begin{aligned}
& j_{e}(\alpha)=\frac{3 \pi v_{F} e}{4 L}\left\{\left[\sin \tilde{\alpha}\left[\lambda+\lambda^{2} \ln (L c)\right]+(1 / 4+\ln 2) \lambda^{2} \sin 2 \tilde{\alpha}\right\}+O\left(\lambda^{3}\right)\right. \\
& j_{o}(\alpha)=\frac{3 \pi v_{F} e}{16 L} \sin 2 \alpha\left[\lambda^{2}+2 \lambda^{3} \ln \left(L c^{\prime}\right)\right]+O\left(\lambda^{4}\right),
\end{aligned}
$$

for $N$ even and odd respectively, where $c$ and $c^{\prime}$ are constants of $\mathrm{O}(1)$ which we have not determined and:

$$
\begin{aligned}
& \tilde{\alpha}=\alpha(N / 2 \text { even }) \\
& \tilde{\alpha}=\alpha+\pi(N / 2 \text { odd }) .
\end{aligned}
$$

$N$ is the number of electrons, including the electron on the quantum dot, $j=0$. At half-filling, $N$ is just the total number of lattice sites, including the origin; $N=L$. The fact that $j$ is $\mathrm{O}(\lambda)$ for $N$ even but not for $N$ odd is easily understood. The unperturbed ground state consists of a partially filled Fermi sea and a decoupled impurity spin. For $N$ even, there are an odd number, $N-1$, of electrons in the Fermi sea. The unpaired electron at the Fermi surface forms a spin singlet with the impurity in first order degenerate perturbation theory. On the other hand, for $N$ odd, there are no unpaired electrons in the non-interacting Fermi sea so it is necessary to go to second order in $\lambda$. A very important property of these results is that, to the order worked, the persistent current only depends on the effective Kondo coupling at the length scale $L: \lambda(L)=\lambda+\lambda^{2} \ln L$. That is, logarithmic divergences are encountered in next to leading order, as is standard for many calculations in the Kondo model, but they only involve $\ln L$, not $\ln T$. The current is finite at $T=0$, for finite $L$. This suggests that the finite size of the ring is acting as an infrared cut-off on the growth of the Kondo coupling. Provided that $\lambda \ln L \ll 1$, the higher order corrections are relatively small and perturbation theory appears trustworthy. This is equivalent to the condition $\xi_{K} \gg L$. We may say that, in this case, the screening cloud doesn't "fit" inside the ring so the Kondo effect (growth of the effective coupling to large values) doesn't occur. We expect that higher order perturbation theory would preserve this property, giving $v_{F} e / L$ times functions of the renormalized coupling at scale $L$ (and the flux) only. This follows because the current obeys a renormalization group equation with zero anomalous dimension. This in turn follows from the fact that the current is conserved $d<j(x)>/ d x=0$ so it can be calculated at a point far from the impurity spin. This scaling form implies, equivalently, that we can write the current as:

$$
j_{e / o}=\frac{e v_{F}}{L} f_{e / o}\left(L / \xi_{K}, \tilde{\alpha}\right)
$$

for even and odd $N$ respectively, where $f_{e}$ and $f_{o}$ are universal scaling functions, depending only on the ratio $L / \xi_{K}$ and not separately on the bare Kondo coupling $\lambda_{0}$ and cut-off $\mathcal{D}$. In the limit $L \gg \xi_{K}$, the persistent current can be calculated using the non-interacting effective low energy Hamiltonian of Eq. (85), i.e. Fermi liquid theory. This gives:

$$
\begin{aligned}
& j_{e}(\alpha)=-\frac{2 e v_{F}}{\pi L}[\tilde{\alpha}-\pi] \quad(N \text { even }) \\
& \left.j_{o}(\alpha)=-\frac{e v_{F}}{\pi L}([\alpha]+[\pi-\pi])\right] \quad(N \text { odd }) .
\end{aligned}
$$


Here

$$
[\alpha] \equiv \alpha(\bmod 2 \pi), \quad|[\alpha]| \leq \pi .
$$

These small $L$ and large $L$ limits are plotted in Fig. (2). Note that the current has the same sign for all $\alpha$ in both limits. Furthermore it has the same period: $2 \pi$ for $N$ even and $\pi$ for $N$ odd. For intermediate lengths, $L$ of order $\xi_{K}$, it is necessary to do a numerical calculation. A combination of exact diagonalization and DMRG [21] supports the scaling behavior of Eq. (93) giving scaling functions that interpolate smoothly, as a function of $\xi_{K} / L$. See. Fig. (3).
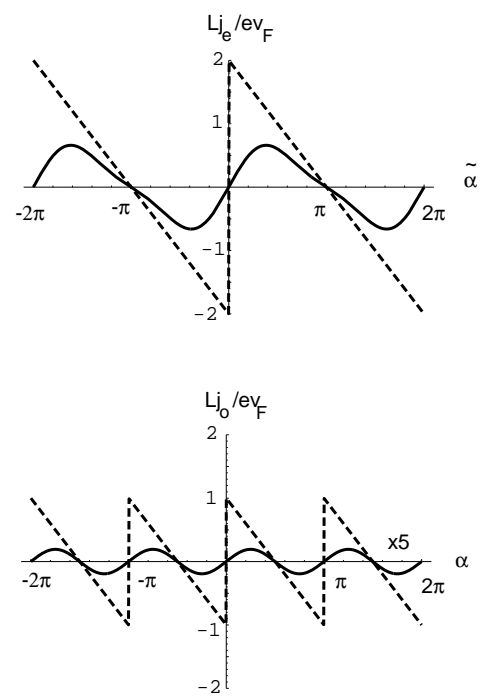

FIG. 2: Persistent current vs. flux for an even or odd number of electrons in the weak coupling limit, for $\xi_{K} / L \approx 50$, from Eq. (91) (solid line) and in the strong coupling limit, $\xi_{K} / L<<1$, from Eq. (95) (dashed line). $j_{o}$ is multiplied $\times 5$ at $\xi_{K} / L=50$ for visibility. The solid lines are obtained from Eq. (91) using the effective coupling $\lambda(L) \approx 1 / \ln \left(\xi_{K} / L\right)$. (From [19].)

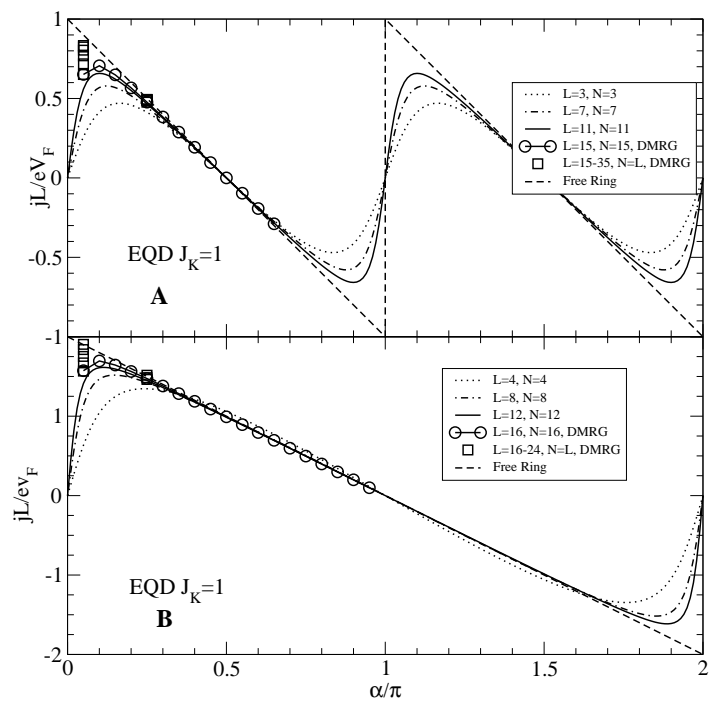

FIG. 3: The current at 1/2-filling for $N=4 p-1$ (A) and $N=4 p$ (B) for the embedded quantum dot. Results 21 are shown for a number of system sizes with $J_{K}=1$ as a function of $\alpha / \pi$. The results for the small system sizes are obtained using exact diagonalization methods while the larger system sizes (circles) have been obtained using DMRG techniques. From Ref. [21]. 


\section{B. Charge steps in a finite length quantum wire terminated by a quantum dot}

Another mesoscopic system which is quite readily analysed theoretically involves a linear quantum wire of finite length with a quatum dot, in the Kondo regime, at one end. [See Fig. (4).] We assume that the system is very weakly tunnel-coupled to an electron reservoir and that a uniform gate voltage, $V_{G}$, is applied to the wire, corresponding to a chemical potential $\mu=-e V_{G}$, in addition to the gates controlling the occupancy of the quantum dot and the tunelling between dot and wire. As $\mu$ is varied, the number of electrons in the system, in its ground state will vary in single steps at particular values of $\mu$. This defines a "charge staircase". See Fig. (5). The width of the steps, with an even or odd number of electrons in the system, is a sensitive indicator of the strength of the effective Kondo coupling. The steps, at discrete values of $\mu$, could be detected by some Coulomb blockade technique. Such measurements are likely to be far easier than measuring persistent currents in closed rings. For simplicity, we treat the wire as having only a single channel - i.e. as being an ideal 1D system. A great advantage of this system is that the charge staircase can be determined very accurately from the Bethe ansatz solution [4,5] of the Kondo model. Remarkably, this remains true, to some extent, even when we include short range Coulomb interactions throughout the wire. In this case we take advantage of the more recent Bethe ansatz solution of an $\mathrm{S}=1 / 2$ Heisenberg antiferromagnet with a weakly coupled spin at one end [22]. We first discuss the case with no Coulomb interactions (apart from those in the quantum dot, leading to the effective Kondo model) and later include bulk Coulomb interactions in the wire.

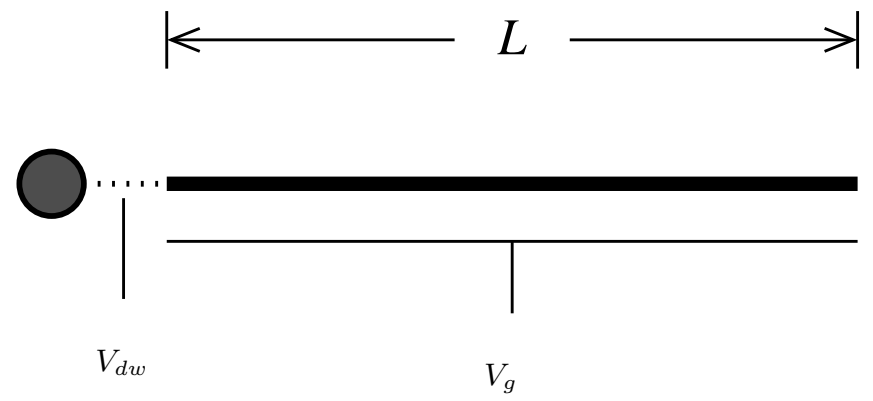

FIG. 4: Possible experimental setup. $V_{d w}$ controls the tunneling $t^{\prime}$ between the small dot (on the left) and the wire and $V_{g}$ varies the chemical potential in the wire.

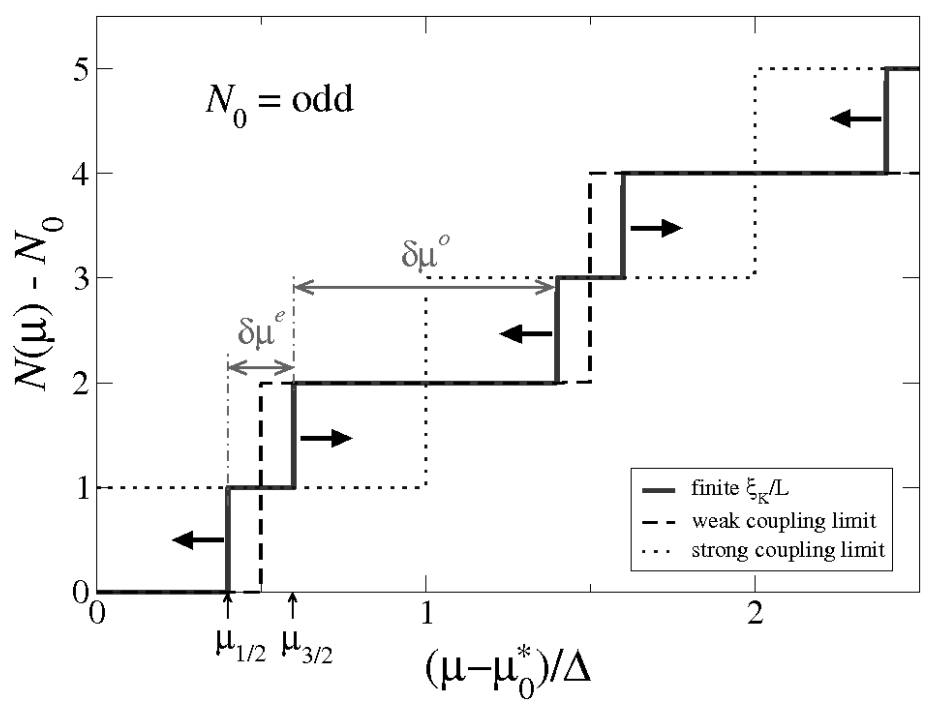

FIG. 5: Charge quantization steps for the wire coupled to a small quantum dot. The arrows indicate the direction in which the single steps move as $\lambda(L)$ grows. 


\section{Interactions in quantum dot only}

Again it is convenient to consider a tight-binding model. In the Kondo limit this is

$$
H=H_{0}+H_{K}
$$

with

$$
H_{0}=-t \sum_{j=1}^{L-2}\left[\left(c_{j \alpha}^{\dagger} c_{j+1, \alpha}+h . c .\right)-\mu c_{j}^{\dagger} c_{j}\right]
$$

and

$$
H_{K}=J c_{1}^{\dagger} \frac{\vec{\sigma}}{2} c_{1} \cdot \vec{S}
$$

Note that this time we have "open" boundary conditions. The chain terminates at site $L-1$. We are interested in how the total electron number in the system, $N$, changes at $T=0$ in the grand canonical ensemble, as we vary $\mu$. We define $N$ to include the single electron on the quantum dot. Considering a small range of $\mu$, the steps with even $N$ all have the same width, as do the steps with odd $N$. We are interested in the ratio $R$ :

$$
R \equiv \frac{\delta \mu^{o}}{\delta \mu^{e}}
$$

where $\delta \mu^{e / o}$ is the width of the interval of chemical potential over which $N$ has a fixed even (odd) value.

The limits of weak and strong Kondo coupling are readily analysed ${ }^{23}$ See Fig. (5). When $J=0$, finite width steps only occur for $N$ odd since the energy levels in the quantum wire are doubly occupied and a single electron resides on the quantum dot. In the opposite limit of strong Kondo coupling, finite width steps only occur for $N$ even. One electron is removed from the Fermi sea to screen the spin and the remaining electrons doubly occup free fermion energy levels, appropriately $\pi / 2$ phase shifted. Thus $R=\infty$ at zero coupling and $R=0$ at strong coupling. Again it is possible to calculate the corrections to these limits at leading order in renormalization group improved perturbation theory at weak coupling and Fermi liquid theory at strong coupling. At weak coupling we find:

$$
\frac{1}{R} \approx(3 / 2)\left[\lambda_{0}+\lambda_{0}^{2} \ln (L / c)\right]+\ldots
$$

As for the persistent current, we see that the length of the quantum wire cuts off the growth of the Kondo coupling, at $T=0$ and we may write:

$$
\frac{1}{R} \rightarrow(3 / 2) \lambda(L) \approx \frac{3}{2 \ln \left(\xi_{K} / L\right)}, \quad\left(L \ll \xi_{K}\right) .
$$

In the other limit, first order perturbation theory in the Fermi liquid interaction of Eq. (15) gives:

$$
R \rightarrow \frac{\pi \xi_{K}}{4 L}, \quad\left(L \gg \xi_{K}\right) .
$$

Again, we see that $R$ appears to be a scaling function of $\xi_{K} / L$ only.

For this case, we can check this scaling hypothesis, and determined the function $R\left(L / \xi_{K}\right)$ to high precision for all $L / \xi_{K}$ by using the Bethe ansatz solution. $\frac{4.5}{5}$ The result is shown in Fig. (6). Excellent agreement is obtained with the asymptotic weak $\left(L \ll \xi_{K}\right)$ and strong $\left(\xi_{K} \ll L\right)$ coupling limits. Weak particle-hole symmetry breaking has a negligible effect on these results, since it simply shifts the entire charge staircase by a constant $\underline{\underline{23}}$

\section{Including Coulomb interactions in the quantum wire}

So far, we have ignored the effect of bulk Coulomb interactions, only considering the Coulomb interaction on the quantum dot, in the Anderson impurity model, which leads to the Kondo interaction. This neglect may be justified in many cases, based on Landau Fermi liquid theory and the fact that the Kondo interaction just involves low energy electron states. However, it is certainly not justified when the Kondo screening takes place in a 1D quantum wire. In this case, Fermi liquid theory breaks down, being replaced by Tomonaga-Luttinger Liquid (TLL) theory. In 1D, 


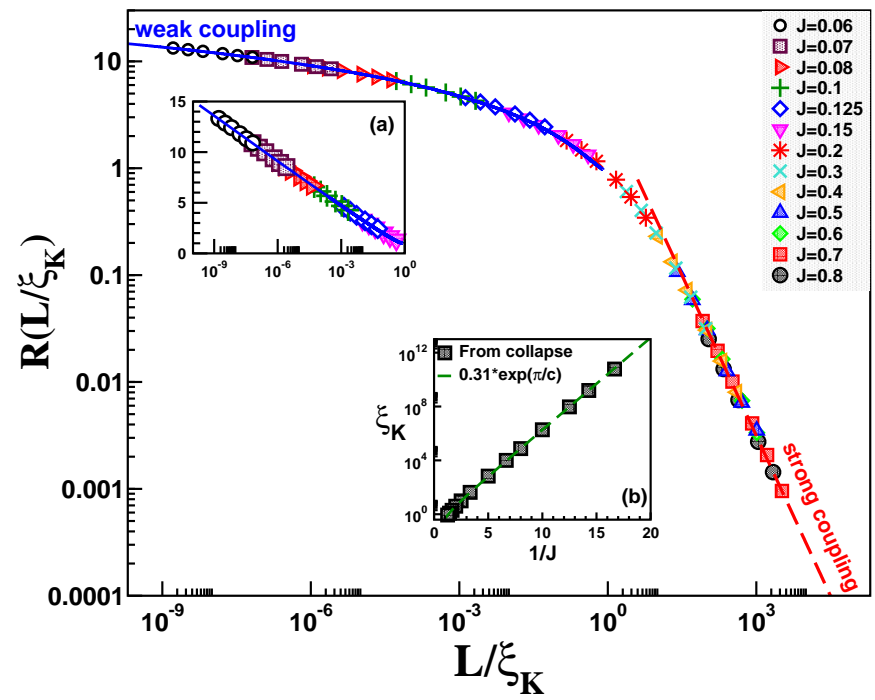

FIG. 6: Universal ratio of even and odd charge steps, $R=\delta \mu^{\circ} / \delta \mu^{\mathrm{e}}$, as a scaling function of $\xi_{K} / L$ from [23]. Bethe ansatz results 23 obtained for various systems sizes $\left(N_{0}=51,101,201,501,1001,2001\right)$ and 13 different values of the Kondo exchange $J$, indicated by different symbols. For each value of $J$, the system lengths $L$ have been rescaled $L \rightarrow L / \xi_{K}(J)$ in order to obtain the best collapse of the data using the strong coupling curve Eq. (103) (dashed red line) as a support for the rest of the collapse. The weak coupling regime for $L \ll \xi_{K}$, enlarged in the inset (a), is described by the weak coupling expansion Eq. (101) with constant $\simeq 0.33$ (continuous blue curve). Inset (b): The Kondo length scale, extracted from the universal data collapse of the main panel (black squares), is described by theexpected exponential dependence on Kondo coupling (dashed green line).

Coulomb interactions lead to major changes in the interaction with an impurity. It turns out however, that these changes are minimized when the magnetic impurity is at the end of a quantum wire, so that the conclusions of the previous sub-sub-section will not be modified too drastically.

We modify the model of Eq. (98) and (99) by adding screened bulk Coulomb interactions. For example, we could consider the Hubbard model with only on-site repulsion:

$$
H_{c}=U \sum_{j} \hat{n}_{j \uparrow} \hat{n}_{j \downarrow}
$$

where $\hat{n}_{j \alpha}$ is the number operator for electrons of $\operatorname{spin} \alpha$ at site $j$. We expect our results to apply to more realistic models, not based on tight-binding approximations and with longer range screened Coulomb interactions. We begin by writing the $1 \mathrm{D}$ electron annihilation operators in terms of left and right movers, similar to what we did for the s-wave projected 3D theory in Eq. (12).

$$
c_{j} \approx e^{i k_{F} j} \psi_{R}(j)+e^{-i k_{F} j} \psi_{L}(j) .
$$

Here $\psi_{R / L}$ vary slowly on the lattice scale, containing Fourier modes of the $c_{j}$ 's in narrow bands around $\pm k_{F}$, appropriate for the low energy effective theory. The Coulomb interactions reduces, in this low energy approximation, to four different terms which can be conveniently written in terms of charge and spin densities (or currents) for left and right movers:

$$
J_{R / L} \equiv \sum_{\alpha} \psi_{R / L \alpha}^{\dagger} \psi_{R / L \alpha}, \quad \vec{J}_{R / L} \equiv \sum_{\alpha \beta} \psi_{R / L \alpha}^{\dagger} \frac{\vec{\sigma}_{\alpha \beta}}{2} \psi_{R / L \beta}
$$

Three of the Coulomb interaction terms are proportional to $\left(J_{L}^{2}+J_{r}^{2}\right), J_{L} J_{R}$ and $\left(\vec{J}_{L}^{2}+\vec{J}_{R}^{2}\right)$. To make further progress, we bosonize the fermions, and introduce separate bosons for the charge and spin degrees of freedom. Upon doing this, the first two interaction terms only involve the charge boson. They change the velocity, $v_{c}$ for charge excitations and also rescale the boson field by the Luttinger parameter for the charge sector, $K_{c}$. The third term changes the velocity, $v_{s}$ for spin excitations. Importantly, all of these terms leave a non-interacting model of two massless bosons, for charge and spin. The fourth Coulomb interaction (exchange) term is written explicitly as:

$$
H_{e x}=-2 \pi g_{0} v_{s} \int d x \vec{J}_{L} \cdot \vec{J}_{R}
$$


where the coupling constant, $g_{0}$, is proportional to the strength of the Coulomb interactions. This can be written entirely in terms of the spin boson operators but corresponds to a non-trivial interaction. The exchange interaction, $g_{0}$ is marginally irrelevant, its effects getting weaker at lower energy, so that asymptotically, free boson behaviour is obtained. Note that this is the opposite to the behaviour of the Kondo coupling which gets stronger at lower energies. We assume the system is not at half-filling so that we can ignore Umklapp interactions.

An important consideration for the charge steps in the wire-dot system we are considering is the boundary conditions on the low energy fields, $\psi_{R / L}$ at $j=1$, the end of the chain next to the quantum dot. In the limit, $J=0$ these are simply:

$$
\psi_{L}(0)+\psi_{R}(0)=0 .
$$

These can be seen, for example, by adding a "phantom site" at $j=0$ and requiring that $c_{0}=0$. Since the low energy fields $\psi_{R / L}$ vary slowly over one lattice spacing, the Kondo interaction can be written in terms of $\vec{J}_{R}(0)$ only:

$$
\begin{aligned}
c_{1} & \approx \psi_{R}(0) e^{i k_{F}}+\psi_{L}(0) e^{-i k_{F}} \approx 2 i \sin k_{F} \psi_{R}(0) \\
c_{1}^{\dagger} \frac{\vec{\sigma}}{2} c_{1} & \approx 4 \sin ^{2} k_{F} \vec{J}_{R}(0) .
\end{aligned}
$$

Since $\vec{J}_{R}$ can be expressed in terms of the spin boson only, the Kondo interaction only involves the spin degrees of freedom. This is not the case if the impurity interacts with the quantum wire far from its end. In that case, we must use the bosonization expression:

$$
\psi_{L}^{\dagger}(j) \frac{\vec{\sigma}}{2} \psi_{R}(j) \approx e^{2 i k_{F} j} \exp \left[i \sqrt{2 \pi K_{c}} \phi_{c}\right] \vec{n}
$$

where $\vec{n}$ can be expressed in terms of the spin boson. Thus, in this situation the Kondo interaction mixes the charge and spin sectors leading to quite different physics. Here, we have deliberately considered the case of the end-coupled spin in order to preserve as much as the physics of the usual Kondo model as possible.

The charge staircase is determined by the ground state energy, as a function of number of particles. In the case where we set to zero both the Kondo interaction, $\lambda_{0}$ and the bulk marginal coupling, $g_{0}$, this is:

$$
E_{0}-\mu N=\frac{\pi v_{c}}{4 K_{c} L}\left(N-N_{0}\right)^{2}+\frac{\pi v_{s}}{L} s^{2}
$$

where $s$ is the spin quantum number of the ground state: 0 for $N$ odd and $1 / 2$ for $N$ even. (Recall that we include the electron on the decoupled quantum dot in our definition of $N$. Here $s$ is the spin of the electrons in the wire, not including the quantum dot.) Including both exchange and Kondo interactions, it is convenient to write:

$$
E_{0}-\mu N=\frac{\pi v_{c}}{4 K_{c} L}\left(N-N_{0}\right)^{2}+\frac{\pi v_{S}}{L} f_{s}\left(\lambda_{0}, g_{0}, L\right)
$$

where $s=0$ or $1 / 2$ for $N$ odd or even and $f_{s}$ are some functions to be determined. Then defining:

$$
f \equiv f_{1 / 2}-f_{0}
$$

it is easily seen that the ratio of step widths is:

$$
\tilde{R}=\frac{1+u f}{1-u f}
$$

where

$$
u \equiv v_{s} K_{c} / v_{c} .
$$

Coulomb interactions made $v_{c}<v_{s} \approx v_{F}$ and also reduce $K_{c}$ from its non-interacting value of 1 . Both these effects reduce $u$ from its non-interacting value of 1 thus bringing $\tilde{R}$ closer to 1 . For cleaved edge overgrowth quantum wires $u \approx .5$ and for carbon nanotubes $u \approx .1$.

In lowest order perturbation theory,

$$
f \approx 1-\frac{3}{2} g(L)-3 \lambda(L), \quad\left(L \ll \xi_{K}\right)
$$


Here we have replaced both the exchange and Kondo couplings by their renormalized values at scale $L$. The exchange interaction lowers the ground state energy when the wire (excluding the quantum dot) has an $s=1 / 2$ ground state, corresponding to $N$ even. The effective exchange coupling is:

$$
g(L) \approx 1 / \ln \left(L / L_{1}\right)
$$

where $L_{1}$ is a short length scale, of order $k_{F}^{-1}$ or smaller. The exchange interactions actually modify the $\beta$-function for the Kondo coupling:

$$
\frac{d \lambda}{d \ln L}=\lambda^{2}+g \lambda+\ldots
$$

(On the other hand, the Kondo interaction doesn't affect the renormalization of $g(L)$ since the Kondo interaction only occurs at the end of the wire and the exchange interaction occurs throughout the wire.) Note that the exchange interaction causes the Kondo coupling to grow faster as the length scale is increased and consequently reduces $\xi_{K}$ for a given bare Kondo coupling:

$$
\xi_{K} \propto \exp \left[\sqrt{a / \lambda_{0}+b}\right]
$$

for some constants $a$ and $b$ depending on $g_{0}$. For long lengths, $L \gg \xi_{K}$, we may again use Fermi liquid theory. Note that the effective exchange coupling, $g(L)$ continues to get smaller as we increase $L$ so we may continue to treat it perturbatively. When $\xi_{K} \ll L$ however, one electron is removed from the Fermi sea to screen the spin of the quantum dot so that the remaining nearly free fermions system has one fewer electron. This switches the $s=0$ and $s=1 / 2$ ground states and changes the sign of the term in $f$ linear in $g(L)$ :

$$
f \approx-1+\frac{3}{2} g(L)+\frac{\pi \xi_{K}}{2 L}, \quad\left(\xi_{K} \ll L\right) .
$$

In general we may write:

$$
f=f\left(\xi_{K} / L, g(L)\right) .
$$

The dependence on $g(L)$ destroys the universal scaling with $L / \xi_{K}$ but in practice this effect can be small since $g(L)$ tends to be small and slowly varying.

Since the Kondo effect for a Luttinger liquid, with the impurity spin end-coupled, only involves the spin degrees of freedom, it is essentially unchanged if the charge degrees of freedom are gapped, as would occur in the Hubbard model at half-filling. In fact, we may take the large $U$ limit at half-filling to obtain the $S=1 / 2$ Heisenberg antiferromagnetic chain with one weakly coupled impurity spin at the end. The only important effect of this large $U$ limit is that the bare exchange coupling, $g_{0}$ becomes $\mathrm{O}(1)$. This impurity model has been solved via Bethe ansatz methods ${ }^{22}$ and this allows an exact calculation ${ }^{23}$ of the function $f\left(L / \xi_{K}, g(L)\right)$ for a particular (large) $g_{0}$. The results are shown, for $L=1000$ and a range of Kondo couplings, in Fig. (7). The expected asymptotic limits, $\pm(1-3 g(1000) / 2)$ are obtained for $L / \xi_{K} \ll 1$ and $\gg 1$. Note that, even for this large bare exchange coupling, the deviations from the model with no bulk Coulomb interactions is quite small. For conducting quantum wires, we expect $g_{0}$ to be considerably smaller and the deviations to be even less. Thus the main effect of the Coulomb interactions is to reduced $\tilde{R}$ due to the parameter $u$ in Eq. (115). If $R$ is the step width ratio in the non-interacting model, we may write it in the interacting case as approximately:

$$
\tilde{R}\left(\xi_{K} / L\right)=\frac{(1-u)+(1+u) R\left(\xi_{K} / L\right)}{(1+u)+(1-u) R\left(\xi_{K} / L\right)} .
$$

This varies from $\tilde{R}\left(\xi_{K} / L\right)=R\left(\xi_{K} / L\right)$ at $u=1$ (no Coulomb interactions) to $\tilde{R}\left(\xi_{K} / L\right)=1$ at $u=0$ (strong Coulomb interactions). Varying $\xi_{K} / L$ at fixed $u, \tilde{R}$ goes from $(1+u) /(1-u)$ at $L \ll \xi_{K}$ to $(1-u) /(1+u)$ at $\xi_{K} \gg L$. Coulomb interactions weaken the even-odd alternation in the step widths but only trivially modify the dependence on $\xi_{K} / L$.

\section{Transport through a finite wire containing a quantum dot}

The previous examples have involved closed systems, making the measurement of the Kondo effect challenging. Most experiments observing the Kondo effect in quantum dots involve transport measurements; the systems are "open", connected to external leads. A natural way of observing finite size effects in a transport measurement is to embed a 


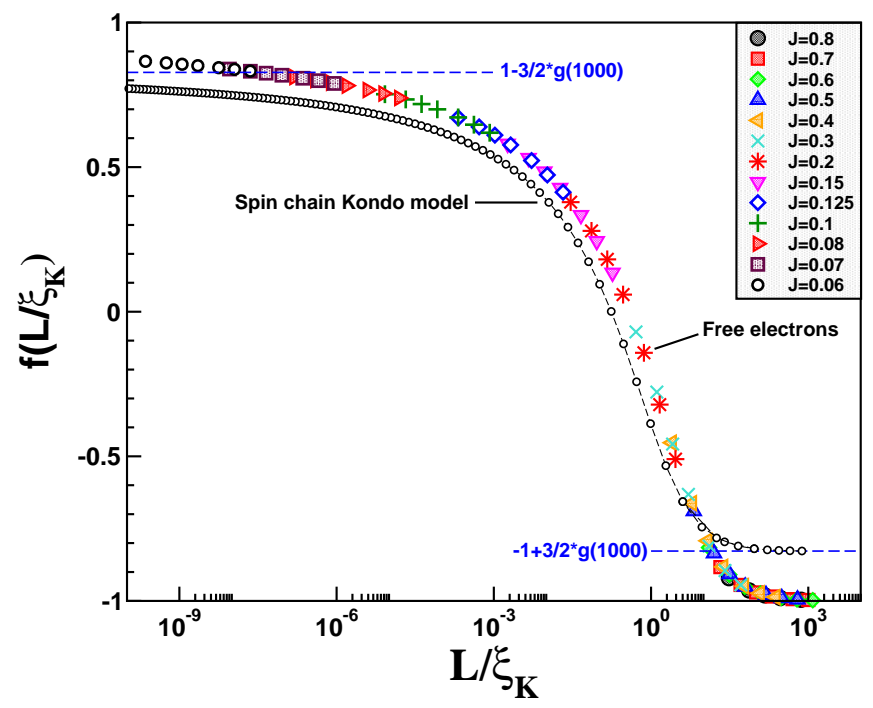

FIG. 7: Effect of the marginally irrelevant bulk operator on the scaling function $f\left(\xi_{K} / L, g(L)\right)$ calculated from the Bethe ansatz from [23]. The symbols correspond to the noninteracting case, $g(L)=0$, as in Fig. 6] The black circles are obtained ${ }^{23}$ with the Bethe ansatz solution of the spin chain Kondo model for $L=1000(g(1000) \simeq 0.115)$.

quantum dot, in the Kondo regime, in a finite length quantum wire which is weakly connected to external leads by tunnel junctions $, \underline{24}, \underline{25}$ as illustrated in Fig. (8). Assuming for simplicity, perfect parity symmetry, the Hamiltonian is:

$$
H=H_{L}+H_{W}+H_{L W}+H_{K}
$$

where $L$ labels the leads, $W$ labels the quantum wire and $K$ the Kondo interaction. Here:

$$
\begin{aligned}
H_{L} & =-t\left[\sum_{j=-\infty}^{-L-2}+\sum_{j=L+1}^{\infty}\right]\left(c_{j}^{\dagger} c_{j+1}+h . c .\right) \\
H_{W} & =-t\left[\sum_{j=-L}^{-2}+\sum_{j=1}^{L-1}\right]\left(c_{j}^{\dagger} c_{j+1}+\text { h.c. }\right)+\epsilon_{W}\left[\sum_{j=-L}^{-1}+\sum_{j=1}^{L}\right] c_{j}^{\dagger} c_{j} \\
H_{L W} & =-t_{L W}\left(c_{-L-1}^{\dagger} c_{-L}+c_{L}^{\dagger} c_{L+1}+h . c .\right) \\
H_{K} & =J\left(c_{-1}^{\dagger}+c_{1}^{\dagger}\right) \frac{\vec{\sigma}}{2}\left(c_{-1}+c_{1}\right) \cdot \vec{S} .
\end{aligned}
$$

Here spin indices are suppressed and we have ignored a potential scattering term, as well as Coulomb interactions in the wire, for simplicity. Note that we have included a chemical potential term, $\epsilon_{W}$ for the wire. When $t_{L W}=0$ the wire + dot system is closed and we expect that the length, $L$, of the wire will act as an infrared cut-off on the growth of the Kondo coupling, leading to scaling in $\xi_{K} / L$ as discussed in the previous two sub-sections. What happens when the wire is connected to the external leads? When $\xi_{K} \ll L$, the Kondo screening cloud fits inside the leads and, at low $T \ll T_{K}$, we therefore expect perfect transmission through the quantum dot. The conductance is then simply that of a finite length non-interacting wire, $W$, connected by tunelling amplitude $t_{W L}$ to the external leads. The conductance is then easily calculated by the Landauer formula which expresses it in terms of the transmission probability for an electron at the Fermi energy to pass through the wire. In this case the Kondo temperature, $T_{K}$ is unaffected by the finite wire length, $L$. On the other hand, when $L \ll \xi_{K}$ the Kondo screening cloud must leak into the external leads. In fact, we might then think of the quantum wire plus dot as acting like a large quantum dot. (But note that, for simplicity, we haven't included an associated charging energy in the Hamiltonian.)

Ignoring the Kondo interactions, the weak tunnelling between wire and leads, $t_{L W}$, leads to a broadening of the discrete spectrum of the wire. That is to say, the local density of states, ocurring in the Kondo interaction, has peaks at the energies given by the discrete spectrum of the wire, with spacing $\Delta=\pi v_{F} / L$ and broadenned by an amount $\delta \propto t_{L W}^{2}$. We assume that $\delta \ll \Delta$. When $L \ll \xi_{K}$, it was found that the conductance of the device depends very sensitively, at low temperatures, on the precise value of $\epsilon_{W}$, which shifts the broadened energy levels of the wire, 


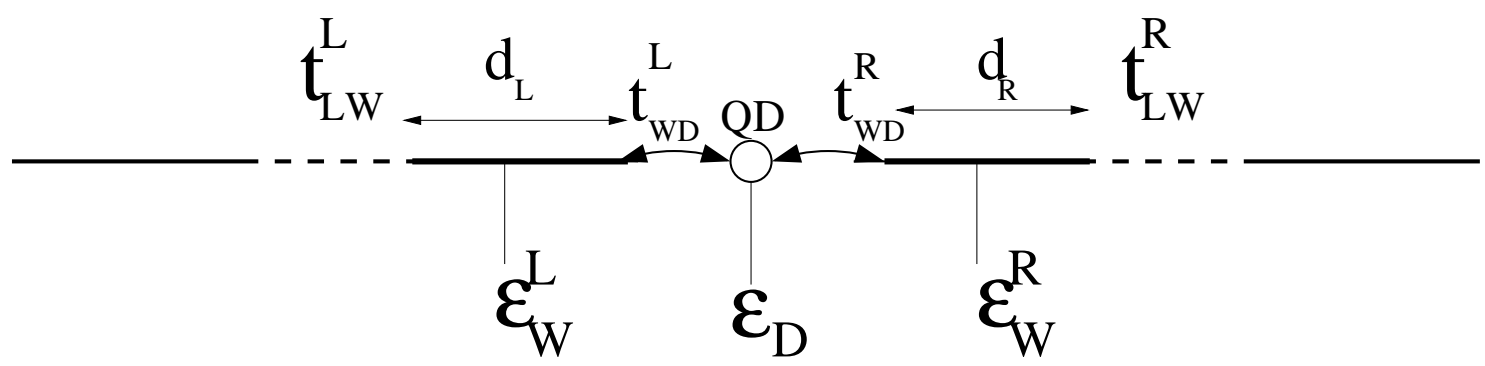

FIG. 8: Quantum dot embedded in finite length quantum wire. $\epsilon_{D}$ and $\epsilon_{W}^{L / R}$ control respectively the dot and wires gate voltage. Here we only consider the parity symmetric case whre all " $L$ " and " $R$ " quantities are equal.

relative to the Fermi energy, moving them on or off resonance. The renormalization of the Kondo coupling may be calculated straightforwardly in second order, using this density of states. Off resonance, there is little renormalization of the Kondo coupling down to exceedingly low energy scales. On resonance, there is again little renormalization until the effective band width is reduced to $\delta$, the width of the resonance peak. At that stage the large density of states leads to a rapid growth of the effective Kondo coupling. In this situation it is convenient to define an effective Kondo temperature, $T_{K}$ which depends on the length, $L$, of the wire and also on the gate voltage applied to the wire, $\epsilon_{W}$. The non-resonant effective Kondo temperature, $T_{K}^{\mathrm{NR}} \approx T_{K}$ when $T_{K} \gg \Delta$, i.e. when the screening cloud fits inside the wire. However, $T^{\mathrm{NR}}$ drops rapidly to zero as $T_{K}$ is reduced below $\Delta$. On the other hand, while the on-resonance Kondo temperature, $T_{K}^{\mathrm{R}}$, also tracks $T_{K}$ when $T \gg \Delta$, as $T_{K}$ is reduced further, $T_{K}^{\mathrm{R}}$ levels off at a value of order $\delta$, the width of the resonance controlled by $t_{L W}$, as illustrated in Fig. (9). Correspondly, the on-resonance conductance through the device, when $\delta \ll T_{K} \ll \Delta$, stays very small down to temperatures of order $\delta$ before shooting up towards the unitary limit, quite unlike the behaviour in the case $\Delta \ll T_{K}$, when the conductance approaches the unitary limit as the temperature is lowered below $T_{K}$. This is illustrated in Fig. (10). The dependence of the low $T \ll \Delta$ conductance on the gate voltage, $\epsilon_{W}$, applied to the wire, is also interesting. When the screening cloud doesn't fit inside the wire, $L \ll \xi_{K}$, there are resonant peaks with spacing in $\epsilon_{W}$ given by the level spacing of the decoupled wires of length $L, \Delta$. On the other hand, when the screening cloud fits inside the wire, $\xi_{K} \ll L$, the resonant peaks in low $T$ conductance are separated by $\Delta / 2$, the level spacing for the doubled wire of length $2 L$.

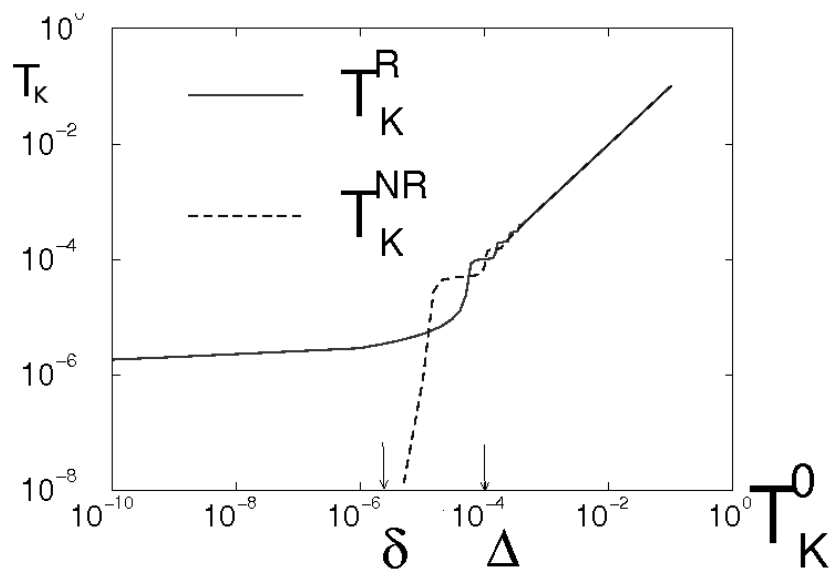

FIG. 9: The curves ${ }^{25}$ represent $T_{K}=f\left(T_{K}^{0}\right)$ in a log-log scale keeping the same values for $\Delta_{n}, \delta_{n}$ for $\epsilon_{W}$ on resonance (plain curve which becomes almost flat at low $T_{K}^{0} \ll \delta_{n}$ ), and $\epsilon_{W}$ off resonance (dashed curve which drops sharply at low $T_{K}^{0}$ ). Both curves coincide at $T_{K}^{0}>\Delta_{n}$. 


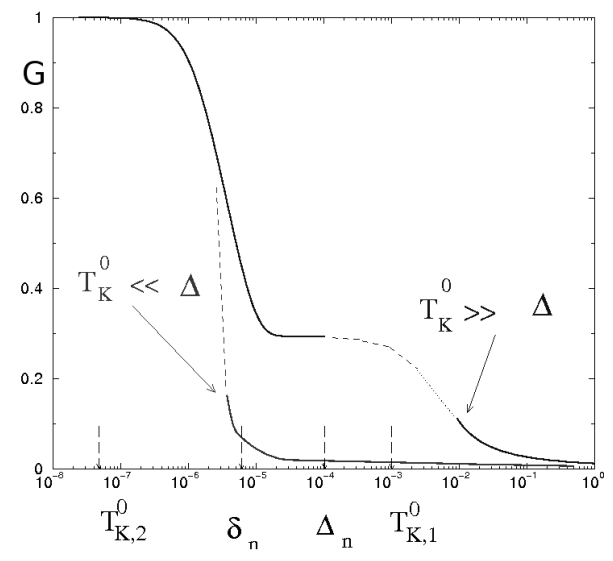

FIG. 10: On-resonance conductance ${ }^{25}$ as a function of temperature in a symmetric device (assuming $\epsilon_{W}$ is on resonance) for both cases $\Delta_{n} \ll T_{K, 1}^{0}$ (right blue curve) and $\Delta_{n} \gg \delta_{n} \gg T_{K, 2}^{0}$ (left red curve), from [25]. The curves in plain style correspond to the perturbative calculations plus the Fermi liquid result for the first case only. We have schematically interpolated these curves (dotted lines) where neither the perturbative nor the Fermi liquid theory applies.

\section{The Kondo Box Model}

In 1999, Thimm, Kroha and van Delft introduced ${ }^{8}$ a model of a Kondo spin in a finite size system, which they referred to as "the Kondo box" model. It behaves quite differently than the models reviewed so far in this article, and here we focus on this difference. The experimental application that these authors discussed, a single magnetic impurity in a disordered ultra-small metallic grain, was rather different than those that we have discussed so far. They adopted a simplified Anderson impurity model in which:

1. All energy levels of the unperturbed system (at $\left.J_{K}=0\right)$ were assumed to be equally spaced in energy near the Fermi energy.

2. All unperturbed states with energies near the Fermi energy were assumed to have equal hybridization with the impurity.

The corresponding Anderson model is:

$$
H=\sum_{i \alpha} \epsilon_{i} c_{i \alpha}^{\dagger} c_{i \alpha}+\epsilon_{0} \sum_{\alpha} d_{\alpha}^{\dagger} d_{\alpha}+U \hat{n}_{d \uparrow} n_{d \downarrow}+v \sum_{i, \alpha}\left(c_{i \alpha}^{\dagger} d_{\alpha}+h . c\right) .
$$

where the $\epsilon_{i}$ are equally spaced. Taking the large $U$ limit, and ignoring potential scattering, the corresponding Kondo model is:

$$
H=\sum_{i \alpha} \epsilon_{i} c_{i \alpha}^{\dagger} c_{i \alpha}+J \sum_{i, j} c_{i}^{\dagger} \frac{\vec{\sigma}}{2} c_{j} \cdot \vec{S}
$$

where $\vec{S}$ is the spin of the magnetic impurity, $\vec{S}=d^{\dagger} \vec{\sigma} d / 2$ and $J=v^{2} / U$. The (uniform) energy level spacing, near the Fermi energy was estimated as:

$$
\Delta=\frac{\pi^{2}}{m k_{F} \mathcal{V}}
$$

where $\mathcal{V}$ is the volume of the grain. Note that in the 1D models considered in this section, the level spacing is proportional to $1 / L$, not $1 / \mathcal{V}$. The behaviour of the Kondo box model follows immediately from the discussion of the 1D models earlier in this section, if we make the corresponding replacement for $\Delta$. As usual, finite size effects become important when $T_{k} \approx \Delta$, the bulk Kondo temperature is of order the level spacing. This implies $\underline{\underline{8}}$ that grains of order $10 \mathrm{~nm}$. in linear dimension are required to see finite size effects, considerably smaller than the estimate of 1 micron in the 1D case. The behaviour of this model seems to contradict the naive Kondo screening cloud picture since there are essentially no finite size effects even when the linear dimension of the grain is much smaller than $v_{F} / T_{K}$.

In fact, the behaviour of this model depends strongly on the assumptions 1 and 2, above, which certainly don't always apply. To make this point it is instructive, as a thought experiment, to consider another model of an ultra-small grain which is far less realistic than even the above one. Imagine that 
1. The grain is completely clean with no disorder

2. The Kondo interaction is short range and spherically symmetric, the standard assumptions in the Kondo model

3. The grain is perfectly spherical with the impurity atom exactly at the centre

In this case, we may expand in spherical harmonics and project onto the s-wave, as usual. The s-wave model is essentially the one studied in sub-section II with the impurity spin at one end of a finite length $1 \mathrm{D}$ wire. Now finite size effects set in when the length $L$ is of order $v_{F} / T_{K}=\xi_{K}$ as discussed above. The essential difference between the two models (or two sets of assumptions) is that in the second case most of the states of the metal have zero (or negligible) coupling to the impurity, whereas in the first case, all states are assumed to have equal coupling. In the second model only the s-wave states couple, a negligible fraction of the total set of states. This happens because the higher harmonic wave-functions all vanish at the origin, where the impurity is assumed to be located. Note also that the Kondo temperature will be much lower for this spherical model since the relevant density of states, appearing in the dimensionless Kondo coupling, scales with the volume in the first case but only as the length in the second.

The behaviour of the second model is very strongly dependent on the assumptions, as can be seen by relaxing only the third of them. Suppose that we allow the surface of the grain to have a non-spherical shape. Then the usual wave-functions, involving products of spherical harmonics and spherical Bessel functions, are no longer eigenstates of the non-interacting model. If we insist on using this basis, then the impurity only interacts with the s-wave as usual. However, the reflections of the spherical harmonic wave-functions off the walls of the grain mix these wavefunctions together. Actually, analogous phenomena occur in the 1D models considered earlier in this section if we take more realistic models of the quantum wires with several channels,$\stackrel{20,25}{2}$ corresponding to transverse wave-functions. Consider, for example, the model in Sec. IIIB of a quantum dot at the end of a finite length quantum wire, which has several channels. The quantum dot hybridizes with some linear combination of channels in the wire. We can always adopt a basis such that one basis state is the one hybridizing with the quantum dot. However, these states are mixed by scattering off the other end of the wire (away from the quantum dot). A simple, intuitive picture, is that the screening cloud wave-function is now a linear superposition of the various channels, or the screening cloud folds back upon itself many times inside the wire, due to the reflections off the ends. If the wire has $N$ channels, its effective length could be as long as $L N$. An important point is that embedding a Kondo spin in a finite system typically destroys one of the key simplifying features of the Kondo model - that the impurity spin only couples to one channel of unperturbed eigenstates (eg. the s-wave). The only case where we can be sure this assumption is still valid is when the finite system is an ideal single channel 1D system.

Depending on various assumptions about the grain shape, magnetic impurity location and disorder, one could imagine the Kondo temperature at which finite size effects set in ranging anywhere from order $\pi v_{F} / L$ to order $\pi^{2} / m k_{F} \mathcal{V}$. In all cases, we expect that the condition for finite size effects to be important is that $T_{K}$ be of order the finite size gap, suitably weighted by the hybridization of the corresponding wave-functions with the impurity. Only in single-channel situations does the simple Kondo screening cloud picture apply.

Note that this complication makes observation of the screening cloud relatively easier in bulk situations where there are no boundaries to mix the hybridizing wave-function with the other channels. However, even there, it seems likely that scattering off other Kondo impurities might have a similar effect.

\section{CONCLUSIONS}

In the standard Kondo model, describing a single magnetic impurity in an infinite host, the length scale $\xi_{K}=v_{F} / T_{K}$ appears as a characteristic crossover length scale for many (perhaps essentially all) physical observables. Some intuition about the nature of the crossover is provided by imagining that, in the ground state, the impurity spin forms a spin singlet with a single conduction electron, whose wave-function is constructed out of states within energy $T_{K}$ of the Fermi surface and hence is spread out over a distance of order $\xi_{K}$. One must be careful to only use this picture at low $T \ll T_{K}$ and even then it sould be used with caution. Naively, we might expect to see weak coupling behaviour, even at $T=0$, at distances $r \ll \xi_{K}$ and strong coupling behaviour, outside the screening cloud, at $r \gg \xi_{K}$. While this seems to apply to the density oscillations and static impurity spin correlations, the first of these expectations turns out be be wrong, or at least to need some modifications, in the case of the Knight shift. The crossover effects at $\xi_{K}$ appear to be very hard to observe experimentally, precisely because they occur at such long distances. In a D-dimensional sample, they are suppressed by a factor of $1 / r^{D}$ (or $1 / r^{D-1}$ ) making their observation more favourable in systems of reduced dimensionality.

In a mesoscopic single-channel $1 \mathrm{D}$ systems with a component of finite length, $L$, there is again typically a cross-over in physical quantities when $\xi_{K}$ is of order $L$. In all cases, weak coupling behaviour occurs when $L \ll \xi_{K}$ so that the screening cloud doesn't "fit" inside the device and strong coupling behaviour occurs when $\xi_{K} \ll L$ so that finite size 
effects become unimportant. Except in ideal circumstances, mesoscopic systems do not have only a single channel. While this is unimportant for the Kondo effect in bulk, it can have important consequences for the observation of the Kondo screening cloud in a mesoscopic device. For an $N$-channel system, the effective length "seen" by the Kondo screening cloud may be as large as $L N$.

Throughout most of this paper we have ignored Coulomb interactions between the conduction electrons, only taking them into account at the magnetic impurity or quantum dot. While this may be justified at low $T$ by Landau's Fermi liquid theory, for $\mathrm{D}=2$ or 3 , it would be necessary for the inelastic scattering length to exceed $\xi_{K}$ for our analyses to apply. In the $\mathrm{D}=1$ case, such interactions are important, even at $T=0$. We analysed them only in a particularly simple case, the quantum dot end-coupled to a single channel quantum wire. While they somewhat reduced effects associated with the Kondo screening cloud, we concluded that the effects are still present and qualitatively unchanged.

Another effect which can interfere with observation of the screening cloud is non-magnetic disorder. We only touched on this briefly in Sec. IIID, is our review of the "Kondo box" model. In addition a finite density of magnetic impurities further complicates the situation.

Throughout essentially all of this review, we have considered only the Kondo model, not the more realistic Anderson impurity model. We expect similar crossover effects to also occur in that case but with another characteristic length scale entering, where the crossover from the free orbital to (unstable) local moment fixed point occurs. The Kondo crossover can only be observed if this other crossover occurs at a significantly shorter length than $\xi_{K}$.

After many years of theoretical and theoretical investigations, the Kondo screening cloud, as predicted by the basic Kondo model, remains undetected experimentally. This is likely due to a combination of experimental difficulties, and limitations of the basic Kondo model. It is to be hoped that further experimental and theoretical progress will eventually bring this long search to a happy conclusion.

\section{Acknowledgements}

I would like to thank all my collaborators in this work. In chronological order: Erik Sørensen, Victor Barzykin, Pascal Simon, Rodrigo Pereria, Nicolas Laflorencie, Bert Halperin, Hubert Saleur and Lazlo Borda. I have also benefited from discussions on this topic with Jan von Delft, Junwu Gan, Nikolai Prokof'ev and Fred Zawadowski. This research was supported in part by NSERC and CIfAR.

1 A.C. Hewson, The Kondo problem to heavy fermions, [Cambridge University Press, Cambridge (1993)].

2 I. Affleck and A.W.W. Ludwig, Nucl. Phys. B360, 641 (1991).

${ }^{3}$ K.G. Wilson, Rev. Mod. Phys. 47, 773 (1975).

${ }^{4}$ N. Andrei, Phys. Rev. Lett. 45, 379 (1980); N. Andrei, K. Furuya and J.H. Lowenstein, Rev. Mod. Phys. 55, 331 (1983).

5 P.B. Weigmann, JETP Lett. 31, 364 (1980).

${ }^{6}$ L. Borda, Phys. Rev. B75, 041307 (2007).

7 I. Affleck, L. Borda and H. Saleur, Phys. Rev. B77, 180404 (2008).

8 W.B. Thimm, J. Kroha and J. von Delft, Phys. Rev. Lett. 82, 2143 (2001).

${ }^{9}$ V. Barzkyin and I. Affleck, Phys. Rev. B61, 6170 (2000).

10 V. Barzykin and I. Affleck, Phys. Rev. Lett. 76, 4959 (1996).

11 V. Barzykin and I. Affleck, Phys. Rev. B57. 432 (1998).

12 J. Gan, J. Phys.:Cond. Mat. 6, 4547 (1994).

13 E. Sørensen and I. Affleck, Phys. Rev. B53, 9153 (1995).

14 J.P. Boyce and C.P. Slichter, Phys. Rev. Lett. 32, 61 (1974); Phys. Rev. B13, 379 (1976).

15 G. Bergman, Phys. Rev. B77, 104401 (2008).

16 F. Mezei and G. Grüner, Phys. Rev. Lett. 29, 1465 (1972).

17 F. Lesage and H. Saleur, J. Phys. A30, L457 (1997).

18 H. Ishii, J. Low Temp. Phys 32, 457 (1978).

19 I. Affleck and P. Simon, Phys. Rev. Lett. 86, 2854 (2001).

20 P. Simon and I. Affleck, Phys. Rev. B64, 085308, 2001.

21 E.S. Sørensen and I. Affleck, Phys. Rev. Lett. 94, 086601 (2005).

${ }^{22}$ H. Frahm and A.A. Zvyagin, J. Phys. 9, 9939 (1997).

23 R.G. Pereira, N. Laflorencie, I. Affleck and B.I. Halperin, Phys. Rev. B77, 125327 (2008).

24 P.S. Cornaglia and C.A. Balseiro, Phys. Rev. B66, 115303 (2002); ibid 174404 (2002); Phys. Rev. Lett. 90, 216801 (2003).

25 P. Simon and I. Affleck, Phys. Rev. Lett. 89, 206602 (2002); Phys. Rev. B68, 115304 (2003). 


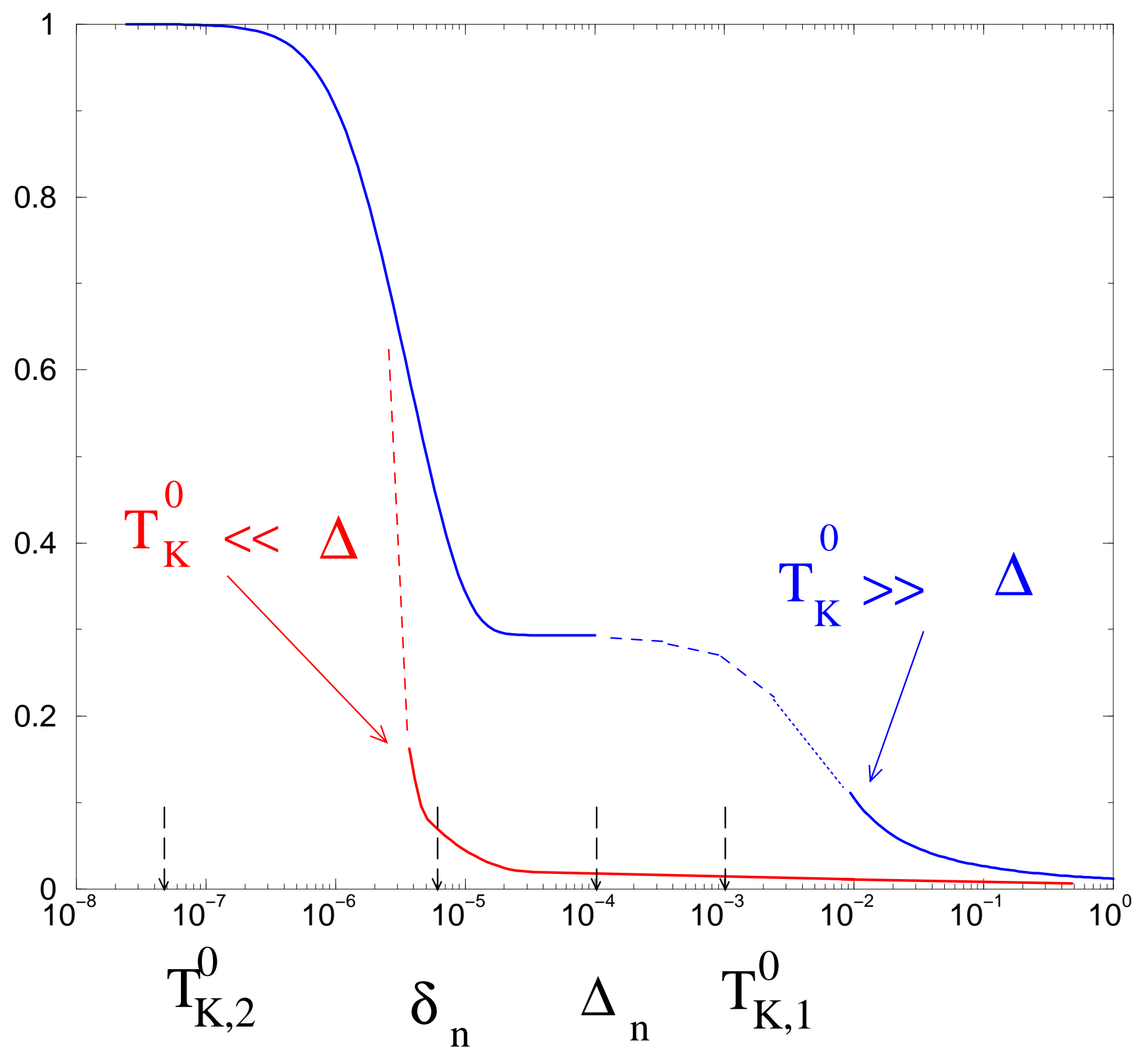




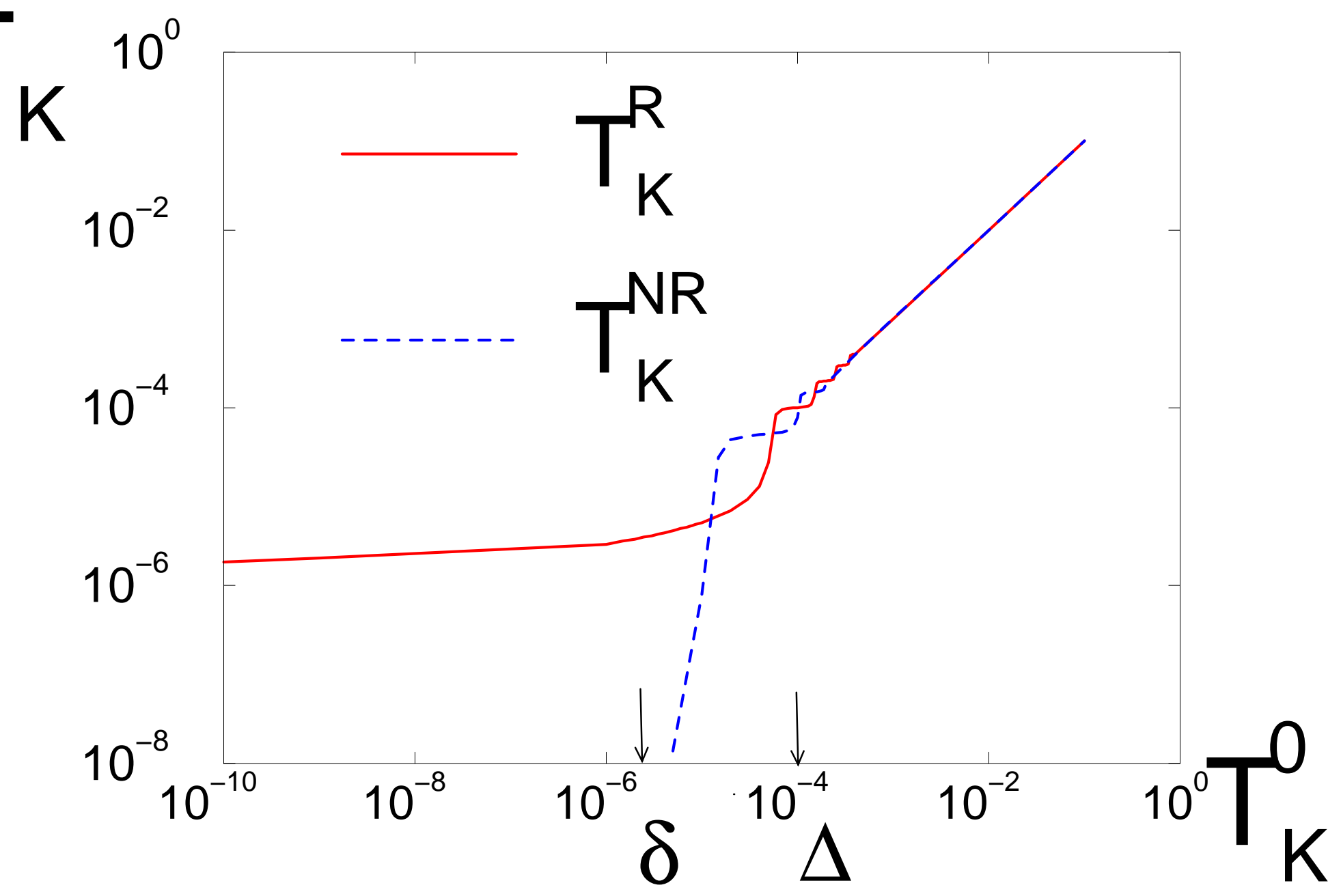

